hep-th/0106128

SLAC-PUB-8862

SU-ITP-01/22

NSF-ITP-01-61

DUKE-CGTP-01-06

\title{
Generating Small Numbers by Tunneling in Multi-Throat Compactifications
}

\author{
Savas Dimopoulos ${ }^{1}$, Shamit Kachru ${ }^{1,2}$, Nemanja Kaloper ${ }^{1,2}$, \\ Albion Lawrence ${ }^{1,3}$, and Eva Silverstein ${ }^{1,2}$ \\ ${ }^{1}$ Department of Physics and SLAC, Stanford University, Stanford, CA 94305/94309 \\ ${ }^{2}$ Institute for Theoretical Physics, University of California, Santa Barbara, CA 93106 \\ ${ }^{3}$ Center for Geometry and Theoretical Physics, Duke University, Durham, NC 27708
}

A generic F-theory compactification containing many D3 branes develops multiple brane throats. The interaction of observers residing inside different throats involves tunneling suppression and, as a result, is very weak. This suggests a new mechanism for generating small numbers in Nature. One application is to the hierarchy problem: large supersymmetry breaking near the unification scale inside a shallow throat causes TeVscale SUSY-breaking inside the standard-model throat. Another application, inspired by nuclear-decay, is in designing naturally long-lived particles: a cold dark matter particle residing near the standard model brane decays to an approximate CFT-state of a longer throat within a Hubble time. This suggests that most of the mass of the universe today could consist of CFT-matter and may soften structure formation at sub-galactic scales. The tunneling calculation demonstrates that the coupling between two throats is dominated by higher dimensional modes and consequently is much larger than a naive application of holography might suggest.

June 2001 


\section{Introduction}

The enormous differences in scales that appear in Nature present a formidable challenge for any unified theory of forces. Grand unification addresses this problem by postulating an energy desert separating the gravitational and the electroweak scale [1]. The supersymmetric version of this picture [2], the supersymmetric standard model (SSM), has had a quantitative success: the unification prediction of the value of the weak mixing angle [2], subsequently confirmed by the LEP and SLC experiments. While this picture is attractive, it leaves many fundamental questions unanswered. There are 125 unexplained parameters, many of them mysteriously small; these include the masses of the three generations of particles and the cosmological constant. String theory provides a natural framework for addressing these questions. Many scenarios for string phenomenology involve localized gauge fields. Perhaps the simplest is the minimal Hořava-Witten theory [3, 1 ; ; other models use "D-brane" defects on which gauge dynamics occurs [5]. A striking possibility emerging from these ingredients is a new explanation the weakness of gravity [6]. These ideas are providing new avenues for exploring physics beyond the Standard Model, and novel mechanisms for explaining small numbers [7,8,9].

Hořava-Witten theory and the perturbative $E_{8} \times E_{8}$ heterotic string [10] have been well studied in calculable, weakly-coupled regimes. In this note we will study string phenomenology in a different calculable regime, which can arise when there are many branes transverse to the compactification manifold $M$. The tension of the branes curves the space around them. The backreaction is proportional to the sum of brane tensions, and therefore to the total number of branes in some region of space. Hence solitary branes have little effect and their neighborhood is nearly flat. Such "dilute gases" of branes are commonly studied in e.g. perturbative string orientifold constructions. In other regimes of couplings where a (super)gravity description is valid, large stacks of branes in the compactification manifold $M$ significantly alter the metric on $M$. The regions of space where the branes reside may be viewed as gravitational funnels, or throats. Examples in this regime arise in F-theory compactifications on elliptic Calabi-Yau fourfolds [11]. From the 4d point of view the geometry is "warped" - the scale factor of the $4 \mathrm{~d}$ metric depends on the distance down the throat. 


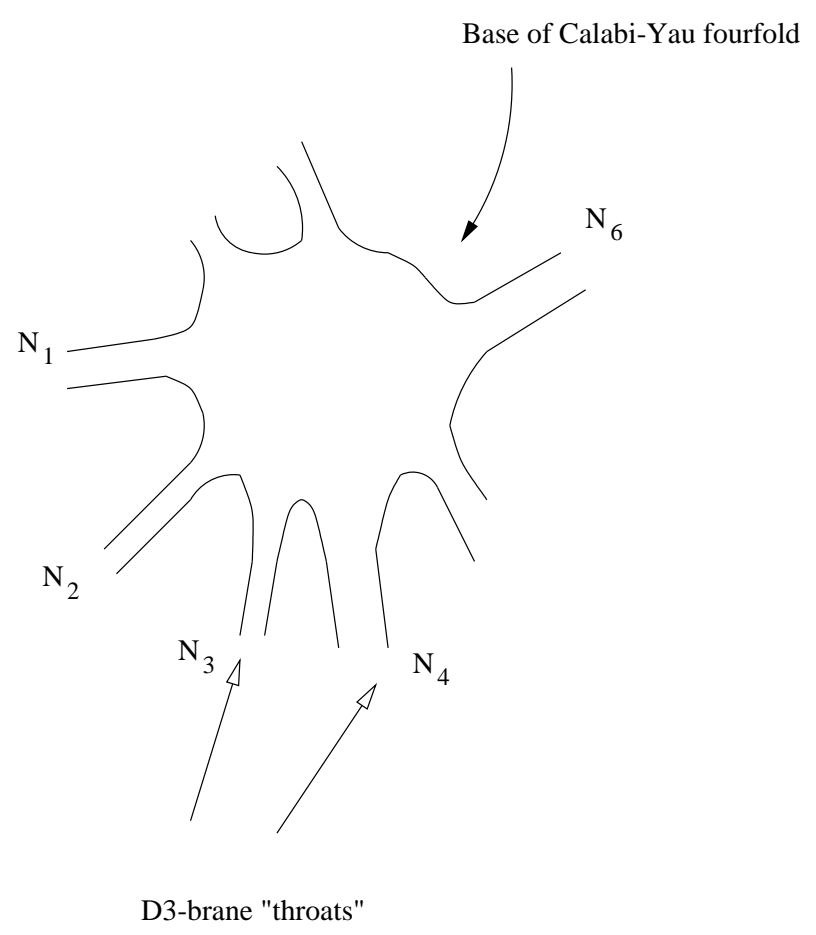

Fig. 1: The Calabi-Yau octopus. $N_{i}$ is the number of branes in a given region.

One class of such scenarios was suggested by H. Verlinde [11], who noted that a wide class of F-theory backgrounds have this property. In F-theory on a Calabi-Yau fourfold $X$, tadpole cancellation accommodates the introduction of

$$
N=\frac{\chi(X)}{24}
$$

D3 branes transverse to $X$ [12]. $N>>1$ is readily achieved for simple choices of $X$. In a suitable limit of couplings, the correct F-theory geometry will involve D3-brane throats glued into the base $\mathcal{B}$ of the elliptically fibered fourfold. A generic possibility is that there are several such throats glued into $\mathcal{B}$; these correspond to different stacks of branes separated on $\mathcal{B}$. The ensuing geometry of the compactification resembles an "octopus," where the legs represent throats arising from brane stacks, as depicted in Fig. 1. The (super)gravity modes in the throat and the low-energy field theory on the branes are dual to each other [13]: the degrees of freedom localized at the ends of the throats are dual to infrared (IR) excitations of the field theory, while the excitations closer to the mouth of the throat are dual to the ultraviolet (UV) degrees of freedom. 
This geometry suggests a new mechanism for generating small numbers in $4 \mathrm{~d}$ physics. The mutual couplings of the IR degrees of freedom residing in different throats are suppressed, as these modes must tunnel through the bulk to communicate. This leads to small couplings between degrees of freedom localized in different throats.

We make this intuition precise in a 5 d toy model of Fig. 1, which appears in Fig. 2. The detailed form of the metric in the throats does not play an important role in our mechanism. The small numbers do not arise from exponential redshifting associated with long throats, but rather from the tunneling suppression emerging due to an effective potential barrier for localized KK modes. Thus the effects we study would persist with generic warped metrics (including those with only power-law warping). However for simplicity and to facilitate a holographic interpretation we will take AdS metrics in the throats of our toy modelt. We will join these brane throats by a "UV brane" playing the role of the bulk of $M$, choosing the scale of physics at the UV brane to be $1 / L$ for simplicity. In general one could choose this scale $M_{U V}$ to be unrelated to either $L$ or $M_{4}$, reflecting the freedom to vary the geometrical moduli near the stacks of branes in the microscopic Calabi-Yau picture. The AdS throats terminate at infrared branes on either side. Such "end-of-the-world" branes mimic a mass gap or be IR-freedom of a generic low energy theory below some scale $\Lambda_{I R}$. We can use the scale-radius duality to cut off the gravitational background at a radius dual to $\Lambda_{I R}$.

We then show explicitly that the KK modes in adjacent throats must tunnel to communicate between different throats, and that this effect generates small numbers.

To gain the insight in the physics of this mechanism one should understand it from the point of view of an effective $4 \mathrm{~d}$ theory. To deduce it, one recalls that the string dynamics far down each throat is dual to a strongly coupled field theory. For AdS warping, a natural guess for any single throat is that the low energy effective theory is a conformal field theory coupled to $4 \mathrm{~d}$ gravity, with a Lagrangian of the form

$$
\mathcal{L}=\mathcal{L}_{C F T}+\sum_{n>0}\left(\frac{1}{M_{4}}\right)^{n} \mathcal{O}_{n}
$$

1 Another option, which would not change the generic physics of the results, would be to use the multi-center D3-brane solution and generalize the computations of Klebanov et al [14, 15] to this case of tunneling between threebrane throats. An intermediate option would be to introduce a flat space between the AdS slices in Figure 2, as in [16]. Here we will stick with the simplest case depicted in Figure 2. 


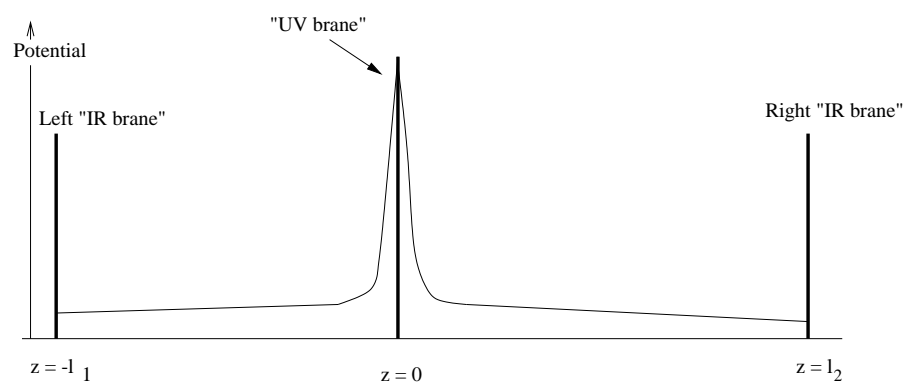

Fig. 2: A cartoon of two throats. The five-dimensional wave equation, for fixed four-momentum, reduces to a one-dimensional Schrödinger equation with a potential barrier as shown. When the throat geometry is anti-de Sitter, the potential is simply the warp factor in conformal coordinates.

here $M_{4}$ is the $4 \mathrm{~d}$ Planck scale and the $\mathcal{O}_{n}$ are irrelevant operators of dimension $4+n$. In the two throat case, one might naively expect the effective theory to be two conformal field theories coupled to each other and to gravity, with an effective Lagrangian of the form

$$
\mathcal{L}_{t o t}=\mathcal{L}_{C F T 1}(\phi)+\mathcal{L}_{C F T 2}(\psi)+\sum_{n>0}\left(\frac{1}{M_{4}}\right)^{n} \mathcal{O}_{n}(\phi, \psi)
$$

here $\phi$ and $\psi$ represent the fields in the two CFTs.

However, in the higher-dimensional gravitational theory the communication between the throats arises from tunneling of the KK modes, and they obey an effective Schrödinger equation which only depends only on the background geometry in the two throats, and not on the $4 \mathrm{~d}$ Planck scale. So in (1.2),(1.3), $M_{4}$ should be replaced by a scale $M_{U V}$ arising from the background geometry. There is a large regime where the geometrical moduli of the compactification are chosen so that $M_{U V} \ll M_{4}$, so communication between distinct throats can be much larger than is suggested by (1.3), as is clear from the Calabi-Yau picture of Figure 1. To see this, one can take a double scaling limit where the volume of $\mathcal{B}$ diverges, while the throats are kept at a fixed relative distance. While $M_{4} \rightarrow \infty$, the communication between the throats persists. This shows that in the $4 \mathrm{~d}$ effective theory, $M_{4}$ arises due to contributions from both the bulk of $\mathcal{B}$ and the throats, as

$$
M_{4}^{2}=M_{5}^{3} L+M_{4,0}^{2}
$$

Thus we can take $M_{4,0}$ to be large while fixing $M_{5}$ and $L$.

Further we find that CFT modes generically mix with $10 \mathrm{~d}$ or $5 \mathrm{~d}$ gravity modes. In the strict near-horizon limit of [13], such modes are non-normalizable and non-fluctuating 
due to the infinite volume of AdS space [17, 18]. In the finite volume setup of Fig. 1, they become normalizable and fluctuating. So the operators $\mathcal{O}_{n}$ are not just made up of operators from the original CFT. We will find that this significantly enhances some phenomenologically interesting processes. This highlights new aspects of the relationship between the physics of RS scenarios and the AdS/CFT correspondence.

The organization of this paper is as follows. In section 2 , we will directly compute the amplitude for an excitation in the left throat to tunnel into the right throat. In section 3, we discuss a potential application of this process in designing candidates for the astrophysical dark matter. In section 4 , we discuss the spectrum of $4 \mathrm{~d}$ excitations in our system and in a closely related system with supersymmetry broken in one of the two throats. We begin an exploration of phenomenological aspects of this "tunneling-mediation" of supersymmetry breaking. In section 5 we present a discussion of the phenomenological aspects of tunneling mediation. We discuss the holographic 4d field theory interpretation of our 5d gravity calculations in section 6. A companion paper, which summarizes the essential physics of this paper but with detailed calculations omitted, appears in [19].

\section{Tunneling and Glueball Decay}

Our generic compactification (Figs 1,2) has a four-dimensional description involving multiple coupled gauge theory sectors. The gauge-invariant excitations in each gauge theory sector will mix to some extent as long as the interactions respect the symmetries of the problem. By the AdS/CFT correspondence, the gauge theory degrees of freedom in the different throats are all comprised of the same bulk degrees of freedom of M theory. At the level of the low-energy gravity modes, this rephrases the question about the mixing of the two gauge theory sectors into a relatively simple double-well potential problem which we now analyze in detail.

For our explicit calculations we will work with the schematic double well background of Figure 2. The metric is given by

$$
d s^{2}=\frac{L^{2}}{(|z|+L)^{2}}\left(\eta_{\mu \nu} d x^{\mu} d x^{\nu}+d z^{2}\right), \quad-l_{1} \leq z \leq l_{2}
$$

in conformally flat coordinates. The proper distances between the UV branes and left and right branes respectively are $R_{1,2}$ where $l_{k}=L e^{R_{k} / L}$. We will assume $l_{1} \geq l_{2}$ throughout the remainder. It will be useful to consider the limit where the UV brane is removed, and 
$4 \mathrm{~d}$ gravity is decoupled. This is readily obtained if we replace $L$ in the denominator of the warp factor in (2.1) by $\zeta L$ and take the limit of the dimensionless parameter $\zeta \rightarrow 0$, removing the cutoff in the $A d S$ space.

The analysis of the KK spectrum is a straightforward generalization of that in [8]. Decompose the transverse traceless mode of the $5 \mathrm{~d}$ graviton as:

$$
h_{\mu \nu}(x, z)=\sqrt{\frac{L}{|z|+L}} e^{i p \cdot x} \psi_{\mu \nu}(z)
$$

and drop the $\mu \nu$ indices. The equation of motion for $\psi$ is:

$$
\psi^{\prime \prime}+\left(m^{2}-\frac{15}{4(|z|+L)^{2}}\right) \psi=0, \quad-l_{1} \leq z \leq l_{2}
$$

where $m^{2}=p^{2}$ is the mass of the $4 D \mathrm{KK}$ mode. $M_{4,5}$ do not appear in this effective Schrödinger equation. The solutions are given in terms of Bessel and Neumann functions in each AdS throat:

$$
\psi(z)=\sqrt{m(|z|+L)}\left(A J_{2}[m(|z|+L)]+B N_{2}[m(|z|+L)]\right)
$$

where $A$ and $B$ are constants.

To compute the tunneling probability through the barrier to the right side, we should impose the proper tunneling boundary conditions. This means that for $z>0$ the wavefunction $\psi$ will be purely outgoing, propagating toward larger $z$, asymptotically approaching $e^{i z / L}$ for large $z$. Since $J_{2}(x)$ and $N_{2}(x)$ behave as $\sqrt{\frac{2}{\pi x}} \cos \left(x-\frac{5}{4} \pi\right)$ and $\sqrt{\frac{2}{\pi x}} \sin \left(x-\frac{5}{4} \pi\right)$ respectively for large argument $x$, the purely outgoing wave to the right must be the Hankel function $H_{2}^{+}(x)=J_{2}(x)+i N_{2}(x)$. On the left, we will have both incoming and reflected waves, and so the wavefunction is a linear combination of both Hankel functions $H_{2}^{ \pm}(x)$. The solution takes the form:

$$
\begin{aligned}
& \psi_{1}=\sqrt{m(|z|+L)}\left(A H_{2}^{+}(m(|z|+L))+B H_{2}^{-}(m(|z|+L))\right), \quad z<0 \\
& \psi_{2}=C \sqrt{m(|z|+L)} H_{2}^{+}(m(|z|+L)), \quad z>0
\end{aligned}
$$

At the UV brane we must impose: continuity of the wavefunction,

$$
\psi_{1}(0)=\psi_{2}(0)
$$


and a matching condition, which comes from perturbing the background geometry junction condition on the UV brane:

$$
\psi_{2}^{\prime}(0)-\psi_{1}^{\prime}(0)=-\frac{3}{L} \psi(0)
$$

These imply:

$$
\begin{aligned}
& B=\frac{H_{2}^{+}(m L)}{H_{2}^{-}(m L)}(C-A) \\
& C=\frac{H_{1}^{-}(m L) H_{2}^{+}(m L)-H_{1}^{+}(m L) H_{2}^{-}(m L)}{H_{1}^{+}(m L) H_{2}^{-}(m L)+H_{1}^{-}(m L) H_{2}^{+}(m L)} A .
\end{aligned}
$$

The transition probability is

$$
P=\frac{\left|\vec{j}_{\text {out }}\right|}{\left|\vec{j}_{\text {in }}\right|}
$$

where $\vec{j}_{i}$ are the currents obtained from the wavefunctions (2.4), (2.7). The $z$-component of the current $j_{z}=-i\left(\psi^{*} \psi^{\prime}-\psi \psi^{*^{\prime}}\right)$ is constant; we can evaluate it at large $|z|$, where the Hankel functions turn into Fourier modes. The tunneling probability is:

$$
P=\left|\frac{H_{1}^{-}(m L) H_{2}^{+}(m L)-H_{1}^{+}(m L) H_{2}^{-}(m L)}{2 H_{1}^{+}(m L) H_{2}^{+}(m L)}\right|^{2}
$$

When the left throat is terminated by an IR brane at $z=-l_{1}$, there is an additional boundary condition,

$$
\psi_{1}^{\prime}=\frac{3}{2\left(l_{1}+L\right)} \psi_{1}\left(l_{1}\right)
$$

Therefore the spectrum of the left-localized states has a gap; the mass $m$ of the tunneling glueball mode is of order $m \sim n / l_{1} \sim \frac{n}{L} e^{-R_{1} / L}$, where $n$ is an integer. Since $m L \sim$ $e^{-R_{1} / L}<<1$, we can expand the Hankel functions appearing in our result (2.9) for small argument and find that:

$$
P \sim m^{4} L^{4} \sim n^{4} e^{-4 R_{1} / L}
$$

Since the incoming flux goes like $1 / l_{1} \sim m / n$, the tunneling rate is given by

$$
\Gamma \sim \frac{\pi^{2}}{16 n} L^{4} m^{5} \sim \frac{\pi^{2} n^{4}}{16} \frac{1}{L} e^{-5 R_{1} / L}
$$

In four-dimensional terms, this is the decay rate of a glueball from the left gauge theory sector into glueballs from the right gauge theory sector. This is important for our discussion of dark matter in $\S 3$. 


\section{Dark Matter}

It is fascinating to contemplate the possibility that the dark matter which constitutes about $90 \%$ of the mass of the universe is described by a CFT, and that we are immersed inside an ocean of scale-free matter.2 In its simplest form this idea is in conflict with observation: CFT matter would have a relativistic equation of state, acting as hot dark matter (HDM); but the large scale structure of the universe suggests that non-relativistic, cold dark matter $(\mathrm{CDM})$ dominates the dynamics of the universe since about $t \sim 10^{4}$ years, when the temperature of the visible matter was $\sim 10 \mathrm{eV}$. A way to bypass this difficulty is to postulate that the universe has, until recently, been dominated by an unstable CDM particle which decays into CFT matter, with a lifetime of order of the age of the universe. This requires a coincidence: the CDM particle must have the right abundance to be a good DM candidate, as well as the right couplings to predominantly decay into CFT matter with a lifetime about as long as the age of the universe. How likely is this coincidence? To answer this we need a concrete model of the CDM, the CFT matter, and their couplings.

A simple model is suggested by the setup of the previous section: Consider the left brane in figure 2 to be at some finite scale, perhaps not far from the electroweak scale $\sim$ $\mathrm{TeV}$, and eliminate the right brane by moving it off to infinity. Imagine that the primordial CDM particles are the lowest-energy, left-localized modes of some five-dimensional field the gravitons or some other bulk particles - located next to the left IR brane. These modes decay by tunneling into the CFT matter residing in the right AdS throat. This setup is sufficiently concrete that it allows a detailed calculation of the abundance and the decay properties of the CDM. The lifetime for the decay of CDM into CFT states is given by Eq. (2.11) and depends on the mass of the particle and the AdS radius.

We can use further data to constrain our model. Several observations suggest that the universe has been dominated, at least until recently, by CDM at the critical density of $\sim 10^{-29} \mathrm{gr} / \mathrm{cm}^{3}$. Under certain conditions, it is possible to infer the necessary microscopic properties that can lead to this abundance. For example, particles that are in thermal and chemical equilibrium - with zero chemical potential- when the universe is at a temperature of order of their mass have an abundance determined by the non-equilibrium dynamics of the expanding universe. Their leftover abundance today can be calculated; it is the result of their failure to totally annihilate with their antiparticles because of the expansion of

2 This possibility has been entertained independently by many physicists, including T. Banks, M. Dine, and J. Maldacena. 
the universe. The energy density, for particles that are non-relativistic when they stop annihilating, is:

$$
\frac{\rho}{\rho_{c}} \simeq \frac{1}{10^{3}<\sigma v>} \frac{1}{M_{P l} \times 2.7 K} \simeq \frac{1}{10^{3}<\sigma v>} \frac{1}{T e V^{2}}
$$

where we have used the fact that $T e V$ is the geometric mean between the Planck scale and the millimeter, $T e V \simeq \sqrt{M_{P l} \times 2.7 K}$. Here $\rho$ is the energy density of these particles at temperature $T$ (which we have set to the present day temperature), $\rho_{c}$ is the critical energy density, and $\sigma$ is the annihilation cross-section of dark matter:

$$
\sigma \simeq \frac{\alpha^{2}}{m^{2}} N
$$

Here $\alpha=g^{2} / 4 \pi$ is the coupling which dominates annihilation, $m$ is the particle's mass, so that their average speed is $v \sim 10^{-3} c$, and $N \sim 100$ is the number of SM particles [20]. We see that a weakly interacting particle, such as the LSP, must weigh about $100 \mathrm{GeV}$ to critically close the universe. However, a strongly interacting particle whose annihilation cross section saturates unitarity will critically close the universe if its mass is about $\mathrm{TeV}$.

Introduce a bulk particle called the "bulky", different than the graviton; let it carry a "Bulk Parity" or B-parity symmetry, under which it changes sign. Decomposing the 5-d bulky into 4-d KK modes, we find that the lightest particle is the massless bulky living deep inside the right throat. On the left we have a quasi-stable bulky which decays to right-bulkies with a lifetime of order of (2.11). Identify the left-brane with the standard model brane on which there are $\sim 100$ particles. B-parity prevents the lightest left-bulky from rapidly decaying into SM particles. On the other hand, pairs of left-bulkies annihilate predominantly into SM particles which reside close by in the fifth dimension. The couplings responsible for bulky annihilation are:

$$
S_{i n t}=\int d^{4} x \sqrt{g_{4}\left(x, z_{b}\right)}\left(g_{5}^{2} \Phi_{b}^{2}(x) \Phi_{B}^{2}\left(x, z_{b}\right)+g_{5} \Phi_{b}(x) \lambda_{b}(x) \lambda_{B}\left(x, z_{b}\right)\right)
$$

where $\Phi_{b}, \lambda_{b}$ are brane scalars and fermions respectively; $\Phi_{B}, \lambda_{B}$ are bulk scalars and fermions at the brane; and $z_{b}$ is the location of the left IR brane in the coordinates of (2.1). In deriving the effective $4 D$ interactions, we normalize the fields so that they have canonical kinetic terms in the $4 D$ metric:

$$
\phi_{4 D i}=a^{d_{i}} \Phi_{i}
$$


Here $a=L /(|z|+L)$ is the scale factor in conformally flat coordinates; $d_{i}$ is the mass dimension of $\phi_{i}$; and $\Phi$ can be a bulk or brane field. Next we perform the mode expansion for the bulk fields,

$$
\begin{aligned}
& \phi_{B}(x, z)=\sum_{m} \phi_{B m}(x) \Psi_{m}(z) \\
& \lambda_{B}(x, z)=\sum_{m} \lambda_{B m}(x) \Psi_{m}(z)
\end{aligned} .
$$

$\Psi_{m}$ are solutions of the Schrödinger equation

$$
\Psi_{m}^{\prime \prime}+\left(m^{2}-V(z)\right) \Psi_{m}=0
$$

with the potential $V$ determined by the spin and bulk couplings of $\phi_{B}$. As a function of $z$, the bulk wavefunctions behave as simple waves in a box. They depend explicitly on the locations of the IR branes in the conformally flat coordinates; and only implicitly on the AdS radius.

Next we expand the action (3.3) in terms of the $4 D$ modes. In addition to the renormalizations above, we must also take into account the overlap between the bulk modes and the brane, $\Psi_{B}\left(y_{b}\right)$ in (3.3). This can be calculated by computing the normalization for the bulk modes which reside between the left IR and the UV brane. Wavefunctions for light, left-localized states are linear combinations of Bessel functions:

$$
\Psi_{m}=\mathcal{N} \sqrt{m(z+L)}\left\{J_{\alpha}[m(z+L)]+(m L)^{\alpha} N_{\alpha}[m(z+L)]\right\} .
$$

Far from the UV brane they asymptote to trigonometric functions. The normalization condition $\int d z\left|\Psi_{m}\right|^{2}=1$ implies that $\mathcal{N}=1 / \sqrt{l}$, where $l$ is the conformal distance between the IR and UV branes. If we take the SM particles to be localized on the left IR brane, the bulk wavefunction is: $\Psi_{m}\left(y_{b}\right) \sim 1 / \sqrt{l}$. The $4 D$ couplings can be determined by the equation:

$$
g_{4}^{2} \phi_{b}^{2} \phi_{B m}^{2}=a^{4}\left(z_{b}\right) g_{5}^{2} \Phi_{b}^{2} \Phi_{B m}^{2}\left(z_{b}\right) .
$$

Since the warp factor on the left IR brane is $a(0) \simeq L / l$,

$$
\begin{aligned}
& \Phi_{b}=\frac{1}{a(0)} \phi_{b}=\frac{l}{L} \phi_{b} \\
& \Phi_{B m}=\frac{\Psi_{m}(0)}{a^{3 / 2}(0)} \phi_{B m} \simeq \frac{l}{L^{3 / 2}} \phi_{B m} .
\end{aligned}
$$

If $g_{5} \simeq 1 / \sqrt{M_{5}}$, then:

$$
g_{4} \simeq \frac{1}{\sqrt{M_{5} L}}
$$


The effective $4 D$ interactions are:

$$
\mathcal{L}_{i n t}=\sum_{m}\left(g_{4}^{2} \phi_{b}^{2}(x) \phi_{B m}^{2}(x)+g_{4} \phi_{b}(x) \lambda_{b}(x) \lambda_{B m}(x)\right)
$$

in canonically normalized field variables.

The annihilation cross section of the bulkies is:

$$
\sigma \simeq N\left(\frac{g_{4}^{2}}{4 \pi}\right)^{2} \frac{1}{m^{2}} \simeq \frac{N}{16 \pi^{2}\left(M_{5} L\right)^{2} m^{2}}
$$

Using (3.1), and the virial velocity of DM particles $v \simeq 10^{-3} c$, the relic density of the bulkies is therefore

$$
\frac{\rho}{\rho_{c}} \simeq 16 \pi^{2} \times 10^{-2}\left(M_{5} L\right)^{2} \frac{m^{2}}{T e V^{2}} .
$$

From this we see that there is a range of parameters for which left bulkies near the electroweak scale can provide the required dark matter density. For example, if $L M_{5} \sim 10$ and $m \sim 100 \mathrm{GeV}$, then the left bulkies close the universe.

Now, using Eq. (1.4) and assuming for simplicity that the Calabi-Yau volume contribution to $M_{4}^{2}$ is comparable to the throats, we find that the value of the AdS radius that leads to a lifetime of the order of the age of the universe is $L^{-1} \sim 10^{14} \mathrm{GeV}$. We should then take $M_{5} \sim 10 / L \sim 10^{15} \mathrm{GeV}$. Finally, assuming down the AdS throat that the extra five dimensions are an Einstein manifold of volume $L^{5}, M_{10} \sim\left(M_{5}^{3} / L^{5}\right)^{1 / 8} \sim 2.3 \times 10^{14} \mathrm{GeV}$. These rough numbers are within a few orders of magnitude of those expected from standard heterotic string model building, and are close to the scale of coupling unification in the MSSM. This rough coincidence suggests that cold dark matter may be decaying into hot CFT dark matter in our epoch; the hot DM then escapes our galaxy. Such a conversion may have observational implications: it can lead to a softening of the dark matter density profile within our galactic halo by spreading it into extragalactic space. (This is the result, for example, of simulations of decaying dark matter performed by Cen [21]).

What about the more economical possibility that the CDM particle is the graviton? In contrast to the bulky, the graviton is not protected by B-parity against decay into SM particles. These decays would be much faster than the slow tunneling into right-gravitons. One way to avoid this is to place the SM sector on the Planck brane in the middle. To see this we consider the $4 D$ effective action for massive gravitons coupled to Standard Model fields, in the linearized approximation. As in Sec. 2, write the graviton fluctuations as: 
$g_{\mu \nu} \rightarrow g_{\mu \nu}+[L /(|z|+L)]^{3 / 2} \Psi_{m} \gamma_{\mu \nu}$, where $\gamma_{\mu \nu}$ is the transverse, traceless $4 D$ tensor and $\Psi_{m}$ is determined by (3.6). The action for $\gamma$ is:

$$
S_{\text {effective }}=\sum_{m}\left\{\int d^{4} x\left(\gamma_{\mu \nu} K^{\mu \nu \alpha \beta}(\partial, m) \gamma_{\alpha \beta}\right)-\int d^{4} x \frac{\Psi_{m}(0)}{M_{5}^{3 / 2}} \gamma_{\mu \nu} T_{S M}^{\mu \nu}\right\}
$$

where $K^{\mu \nu \alpha \beta}(\partial, m)$ is the inverse propagator for massive $4 D$ gravitons, $T^{\mu \nu}$ is the stressenergy tensor for SM fields and we have again normalized the fields to have canonical kinetic terms on the UV brane.

The KK graviton wavefunctions are described by Bessel functions as explained above, with index $\alpha=2$. Far from the UV brane they are approximated by ordinary trigonometric functions, and hence as before are properly normalized by $\sim 1 / \sqrt{l}$. On the other hand, near the UV brane these wave functions are dominated by the Neumann functions, of order

$$
\Psi(0) \sim \sqrt{m(z+L)} m^{2} L^{2} N_{2}[m(z+L)] / \sqrt{l} \sim \sqrt{m L / l} .
$$

This provides additional suppressions for the couplings. Because $a(0)=1$ on the UV brane, the coupling of the low-lying left-localized massive $5 D$ gravitons to matter on the $\mathrm{UV}$ brane is $g \sim \sqrt{(m L / l)} / M_{5}^{3 / 2} \gamma_{\mu \nu} T^{\mu \nu}$. Hence the decay rate into SM particles is given by

$$
\Gamma_{C D M \rightarrow S M} \sim N g^{2} m^{3} \sim \frac{m^{4} L^{4}}{l\left(M_{5} L\right)^{3}} N=\frac{\Gamma_{C D M \rightarrow C F T}}{\left(M_{5} L\right)^{3}} N .
$$

Since the rank of the gauge group in the dual CFT is $\mathcal{N}^{2}=\left(M_{5} L\right)^{3}$, the branching ratio is:

$$
\frac{\Gamma_{C D M \rightarrow S M}}{\Gamma_{C D M \rightarrow C F T}}=\frac{N}{\mathcal{N}^{2}} .
$$

Using the numbers above, $M_{5} L \sim 10$ implies $\mathcal{N} \sim 1000$, and since (MS)SM has on the order of a 100 flavors, we find $\Gamma_{C D M \rightarrow S M} / \Gamma_{C D M \rightarrow C F T} \sim 0.1$. Hence when SM is localized on the UV brane, the main decay mode of CDM is into CFT hot dark matter, simply because of the universality of their couplings via the stress-energy tensors and the large number of degrees of freedom in the CFT.

However, since there are no particles on the left-brane, the left-graviton has a small annihilation cross-section into the now distant SM particles or the right-gravitons. To determine this, we need the graviton-matter couplings which are quadratic in the graviton field. It is clear that in terms of the graviton field $\gamma_{\mu \nu}$ defined previously, these are

$$
S_{i n t}=\sum_{m}\left\{\int d^{4} x \sqrt{g_{4}} \frac{\Psi_{m}^{2}(0)}{M_{5}^{3}}\left(\mathcal{O}(1) \gamma_{\lambda \mu} \gamma^{\lambda}{ }_{\nu} T_{S M}^{\mu \nu}+\mathcal{O}(1) \gamma_{\mu \nu} \gamma^{\mu \nu} T_{S M}\right)\right\}
$$


where $T_{\mu \nu}$ and $T$ are the brane-localized SM stress-energy tensor and its trace. Since $\Psi_{m}(0) \sim \sqrt{m L / l}$, the quadratic graviton coupling is

$$
g \sim \frac{m L}{l M_{5}^{3}}
$$

and because it has dimension -2 , the annihilation cross section for the process $\gamma \gamma \rightarrow S M$ is

$$
\sigma \sim g^{2} m^{2} \sim \frac{m^{4}}{l^{2} M_{5}^{6}} L^{2}
$$

For the lightest KK gravitons, $m \sim 1 / l$ and hence $\sigma \sim\left(m / M_{5}\right)^{6} L^{2}$. This cross section is so small that when the gravitons are relativistic their annihilation ceases, so that their "freezeout" abundance is comparable to that of photons. This means that they are not cold but hot dark matter which is disfavored by the observed structure formation at small scales. After freezeout the gravitons are decoupled from the rest of the particles whereas the photons continue to get "heated-up" at every threshold and, as a result, the photon abundance today would be one to two orders of magnitude larger than that of gravitons. Therefore in order that left-gravitons close the universe today, they must weigh about $10 \mathrm{eV}$. So the conformal distance between the left IR brane and the UV brane is $l \sim$ $(10 \mathrm{eV})^{-1}$. Furthermore, in order that its lifetime be the Hubble time the AdS radius must be $L \sim G e V^{-1}$, and so $M_{5} \sim 10^{13} \mathrm{GeV}$.

An alternative possibility would be to postulate a time dependence in the distance of the left brane from the UV brane which allows for the annihilation cross section of the gravitons to be large at some early epoch. This, or any other mechanism enhancing the early graviton annihilation cross section could allow for heavy CDM gravitons. However, this amounts in practice to giving up calculability and simply postulating the existence of a mechanism giving rise to the correct CDM abundance for $\sim T e V$ gravitons.

Cosmological models with standard cold dark matter consisting of weakly interacting massive particles (WIMPS) successfully account for the large scale structure of the universe at extragalactic scales. However, there have been suggestions of a number of problems at subgalactic scales, associated with an apparent excess of small scale structure. Simulations predict cuspy halo density profile at subgalactic scales which give rise to galaxy and cluster cores that appear to be overly dense. They also predict a large number of subhalos which are disfavored by the scarcity of satellite galaxies in clusters. There have been several proposals to account for these discrepancies including collisional dark matter [22], fluid 
DM [23], decaying DM [21] and so on. The common theme is the presence of a mechanism that spreads the localized CDM energy into a larger region of space.

Our proposal is similar to these ideas and its implications for structure formation are close to the scenario of [21] where the CDM particle decays to light, invisible particles neutrinos, for example - which escape our galaxy. There it was shown by detailed computer simulations that if a significant fraction (e.g., one-half) of the CDM decays into neutrinos, then at least some of the problems with excess small-scale structure are avoided. The only difference in our case is that the decay product is CFT-matter instead of neutrinos. A potential difficulty of this scenario is that it predicts that younger galaxies should be heavier by an amount which may be too large 3 .

A variation of this scenario is to add a right IR brane. If the characteristic length scale of this brane is larger than a galactic halo $\sim M p c$ the previous discussion is not significantly altered: we are again dealing with an approximate CFT. If the scale of the right brane is less than a galactic halo then the IR dynamics of confinement sets in before the right localized gravitons escape our halo and may prevent them from leaving it. The detailed computation of sub-galactic structure formation is expected to be involved in this case. After all, even for the simple dissipationless cold dark matter structure computation gave surprising results, such as the excess subgalactic structure we are attempting to undo. Nevertheless, it is clear that the possibility of confining the right-gluons within the galaxy will eliminate the problem of heavier young galaxies mentioned earlier. Still, depending on the scale of confinement, the decaying CDM model suggests that faraway galaxies should have denser and more compact cores because they are younger.

\section{Spectrum and Tunneling-Mediated Supersymmetry Breaking}

Low-energy SUSY is one of the most attractive scenarios for physics beyond the Standard Model, because it stabilizes scalar masses at the SUSY breaking scale [2]. We must still explain the origin of the low SUSY breaking scale and the 125 physical parameters in the MSSM [24]. The standard lore is to postulate that supersymmetry is broken in a hidden sector, and that the breaking communicated to the visible sector via some messenger fields. There is a variety of SUSY breaking mediation mechanisms such as gravity [25,26,27], gauge [28,29], anomaly [30], gaugino [31] mediation, in hidden sector scenarios.

3 We thank Paul Steinhardt for pointing this out. 
In brane world models, supersymmetry can be broken on a brane located "elsewhere" in the extra dimensions [3] [32]. The warped compactifications provide a new calculable regime for this kind of idea. Specifically tunneling effects between brane throats provide a new mechanism for generating a small SUSY breaking scale. Here we consider the following scenario. We take both IR branes to be close to the UV brane. SUSY is broken on the left IR brane via soft mass-like terms for bulk modes. This induces SUSY breaking mass splittings on the right IR brane, where the SSM resides. A hierarchy is generated because SUSY breaking at a high scale on the left IR brane induces small SUSY breaking mass splittings in the SSM, due to tunnelling supression. We choose the distance between the right IR brane and the UV brane, as well as the bulk parameters $M_{5}$ and $L$, to be near the GUT scale $M_{G U T} \sim 10^{16} \mathrm{GeV}$. This ensures that the cutoff on the right SSM brane is $M_{G U T}$; consequently supersymmetric gauge coupling unification [2, 33] can be preserved. We find that there is a regime where the $5 D$ tunneling effects could be the dominant channel for transmitting SUSY breaking to the visible sector.

There may be different sources of SUSY breaking in the distant throat. The simplest way to break supersymmetry is by considering a bulk hypermultiplet with a massless scalar $\phi$, and breaking supersymmetry explicitly with a mass-like term for $\phi$. We will consider this example first, and later will consider a more realistic but involved case when SUSY is broken by Majorana-like mass terms for bulk fermions, which is necessary to generate gaugino masses.

In order to separate the effects of hierarchy generation by warp factor scaling as in [8] from the effects of the tunneling, we consider two cases:

1) Direct SUSY breaking: We live on the left IR brane at $z=-l_{1}$, and SUSY is broken in the left throat. The effects of the right throat on SUSY breaking are negligible. This case provides a contrast for the next case, which is our main focus.

2) Tunneling mediation: In the two-throat geometry of Figure 2, supersymmetry is broken in the left throat, and our world is right IR brane at $z=l_{2}$.

We then compute the mass splittings these effects induce on particles localized on the relevant branes: the left IR brane for case 1), and the right IR brane for case 2).

We discuss two (qualitatively different) types of supersymmetry breaking: Type A) SUSY is broken everywhere in the left throat, for example by taking a IIB string theory background of the form $A d S_{5} \times X$, where $X$ is an Einstein manifold or deformation thereof which breaks supersymmetry (simple examples, from orbifolds or RG flows starting from $A d S_{5} \times S^{5}$, appear in 34 ). We model this with a step function mass squared for bulk 
particles $m_{0}^{2}+\bar{q}^{2}$ for $z<0$ and $m_{0}^{2}$ for $z>0$, where only the $\bar{q}^{2}$ piece breaks supersymmetry (i.e. it is zero for the superpartner.)

Type B) Supersymmetry-breaking is localized on the left IR brane. In the AdS/CFT correspondence, this corresponds to a case where the gauge theory dual to closed strings in the left throat dynamically breaks supersymmetry, and so is supersymmetric down to a low scale. We model this with a localized, $\delta$-function mass on the left brane.

In $\S 4.1$ we consider the spectra of bulk modes in the presence of the SUSY breaking terms of types A) and B). We also discuss the decomposition into modes localized in the left and right throat, and delineate the approximations made in the process. In $\S 4.2$ we discuss type A) SUSY breaking in detail and determine the couplings of the left and right localized bulk modes to the right IR brane. In $\S 4.3$, we redo this for type B) SUSY breaking. In $\S 4.4$ we calculate the splittings of superpartners on the right IR brane. We also find the splittings on the left IR brane in the various cases, to compare type 1) and type 2) SUSY breaking.

These calculations illustrate how SM matter fields obtain their SUSY breaking masses. To understand gaugino masses, we must break R-symmetry, and we give an example in $§ 4.5$. We discuss the parameter values of the $5 \mathrm{~d}$ model which would lead to phenomenologically interesting and viable SUSY breaking in $\S 5$.

\subsection{Spectra of Bulk Modes with Broken SUSY}

We study a scalar field $\phi$ with supersymmetric bulk mass $m_{0}$ propagating in the background metric (2.1). The IR branes function as orbifold planes for the purposes of defining boundary conditions for bulk fields, as in [3, 8]. We will assume that $l_{1}>l_{2}$ both for model building purposes and in order to use nondegenerate perturbation theory for determining the spectra of bulk modes. If we had taken $l_{1}=l_{2}$ instead, the low-lying modes would have had nearly degenerate states, even or odd under $z \rightarrow-z$. However $l_{1}>l_{2}$ automatically selects the left- and the right-localized states as the natural basis of bulk modes. We can then immediately determine the mass splitting for all bulk states, avoiding the complications arising from the near-degeneracy of symmetric and antisymmetric modes when $l_{1}=l_{2}$.

With SUSY breaking mass terms $M_{0}^{2}=m_{0}^{2}+\bar{q}^{2} \theta\left(-\left(z+l_{1}\right)\right)$ and $2 q$ (where $\bar{q}$ parameterizes the type A) breaking and $2 q$ the type B) breaking), the scalar equation is

$$
\frac{1}{\sqrt{-G}} \partial_{a}\left(\sqrt{-G} G^{a b} \partial_{b}\right) \phi=\left(M_{0}^{2}+2 q \sqrt{\frac{G_{4}}{G}} \delta\left(z+l_{1}\right)\right) \phi,
$$


where $G$ is the $5 D$ metric, $G_{4}$ is the induced $4 D$ metric and the indices $a, b$ run over all 5 dimensions. Making the KK ansatz appropriate for modes of fixed $4 \mathrm{~d}$ mass $m$ (which satisfy the $4 D$ Klein-Gordon equation $\left.\partial_{4}^{2} \phi=m^{2} \phi\right)$, and defining

$$
\psi=\left(\frac{L}{|z|+L}\right)^{3 / 2} \phi
$$

one finds that $\psi$ satisfies Bessel's equation

$$
\psi^{\prime \prime}+\left(m^{2}-\frac{15+4 M_{0}^{2} L^{2}}{4(|z|+L)^{2}}-2 q \frac{L}{l_{1}+L} \delta\left(z+l_{1}\right)\right) \psi=0,
$$

where we take the supersymmetric brane-localized terms to vanish, since they do not alter the conclusions qualitatively. Using the new variable

$$
w=m(|z|+L)
$$

we can rewrite the mode equation (4.3) in the bulk as

$$
\frac{d^{2} \psi}{d w^{2}}+\left(1-\frac{15+4 M_{0}^{2} L^{2}}{4 w^{2}}\right) \psi=0
$$

The solutions are

$$
\begin{array}{ll}
\psi(w)=\psi_{L}(w)=\sqrt{w}\left(a_{L} J_{\nu_{L}}(w)+b_{L} J_{-\nu_{L}}(w)\right), & z<0 \\
\psi(w)=\psi_{R}(w)=\sqrt{w}\left(a_{R} J_{\nu_{R}}(w)+b_{R} J_{-\nu_{R}}(w)\right), \quad z>0
\end{array}
$$

where $J_{\alpha}$ are the Bessel functions of index $\alpha$, and where

$$
\nu=\sqrt{4+M_{0}^{2} L^{2}}
$$

Note that the indices of the Bessel functions differ in the two throats, due to the step function mass. We have taken $\nu_{L, R}$ to be noninteger numbers to simplify the calculations; otherwise $J_{-\alpha}$ should be replaced by Neumann functions $N_{\alpha}$. This assumption will not affect our final result.

The wavefunctions satisfy the boundary conditions

$$
\begin{array}{ll}
\psi^{\prime}\left(-l_{1}\right)=q \frac{L}{l_{1}+L} \psi\left(-l_{1}\right), & \text { left IR brane } \\
\psi\left(0_{+}\right)=\psi\left(0_{-}\right), \quad \psi^{\prime}\left(0_{+}\right)=\psi^{\prime}\left(0_{-}\right), & \text {UV brane } \\
\psi^{\prime}\left(l_{2}\right)=0, & \text { right IR brane }
\end{array}
$$


The first equation comes from the $\delta$-function SUSY breaking mass term on the left IR brane. In the absence of this term, the wavefunction would satisfy Neumann boundary conditions on the left IR brane, as it does on the supersymmetric IR brane on the right. In general, the boundary conditions corresponding to the supersymmetric limit may be different, but that does not change our qualitative conclusions.

The solutions depend on five integration parameters $a_{L, R}, b_{L, R}, m$. Removing the overall scale of the wavefunction, and imposing the four boundary conditions at $z=0$ and $z_{1}=-l_{1}, z_{2}=l_{2}(4.9)$, we find a discrete tower of solutions for $m$. We now discuss separately the cases when supersymmetry is broken in the whole left throat and when it is broken only on the left IR brane.

\subsection{SUSY Breaking in the Left Throat}

Let us first set $\bar{q} \neq 0, q=0$. The superpartner has mass $m_{0}$, so SUSY is unbroken in the right throat. We compute the mass splittings in the 4d Kaluza-Klein tower and the effective $4 \mathrm{~d}$ couplings to the fields on the IR branes.

For the case $\bar{q}<<m_{0}$,

$$
\begin{aligned}
& \nu_{R}=\nu=\sqrt{4+m_{0}^{2} L^{2}} \\
& \nu_{L}=\sqrt{4+M_{0}^{2} L^{2}} \sim \nu+\frac{\bar{q}^{2} L^{2}}{2 \nu}
\end{aligned}
$$

This approximation is very good because of the quadratic dependence of $\nu_{L}$ on $\bar{q}$.

Substituting the solutions (4.6), (4.7) into the eqs. (4.9) gives

$$
\begin{aligned}
b_{R} & =\alpha a_{R}+\beta a_{L} & b_{L} & =\bar{\beta} a_{R}+\bar{\alpha} a_{L} \\
b_{R} & =\gamma a_{R} & b_{L} & =\bar{\gamma} a_{L}
\end{aligned}
$$


where the coefficients are

$$
\begin{aligned}
& \alpha=\frac{\frac{J_{\nu_{R}-1}(m L)-\left(\nu_{R}-1 / 2\right) J_{\nu_{R}}(m L) / m L}{J_{-\nu_{L}+1}(m L)+\left(\nu_{L}-1 / 2\right) J_{-\nu_{L}}(m L) / m L}-\frac{J_{\nu_{R}}(m L)}{J_{-\nu_{L}}(m L)}}{\frac{J_{-\nu_{R}+1}(m L)+\left(\nu_{R}-1 / 2\right) J_{-\nu_{R}}(m L) / m L}{J_{-\nu_{L}+1}(m L)+\left(\nu_{L}-1 / 2\right) J_{-\nu_{L}}(m L) / m L}+\frac{J_{-\nu_{R}}(m L)}{J_{-\nu_{L}}(m L)}} \\
& \beta=\frac{\frac{J_{\nu_{L}-1}(m L)-\left(\nu_{L}-1 / 2\right) J_{\nu_{L}}(m L) / m L}{J_{-\nu_{L}+1}(m L)+\left(\nu_{L}-1 / 2\right) J_{-\nu_{L}}(m L) / m L}+\frac{J_{\nu_{L}}(m L)}{J_{-\nu_{L}}(m L)}}{\frac{J_{-\nu_{R}+1}(m L)+\left(\nu_{R}-1 / 2\right) J_{-\nu_{R}}(m L) / m L}{J_{-\nu_{L}+1}(m L)+\left(\nu_{L}-1 / 2\right) J_{-\nu_{L}}(m L) / m L}+\frac{J_{-\nu_{R}}(m L)}{J_{-\nu_{L}}(m L)}} \\
& \bar{\alpha}=\frac{\frac{J_{\nu_{L}-1}(m L)-\left(\nu_{L}-1 / 2\right) J_{\nu_{L}}(m L) / m L}{J_{-\nu_{R}+1}(m L)+\left(\nu_{R}-1 / 2\right) J_{-\nu_{R}}(m L) / m L}-\frac{J_{\nu_{L}}(m L)}{J_{-\nu_{R}}(m L)}}{\frac{J_{-\nu_{L}+1}(m L)+\left(\nu_{L}-1 / 2\right) J_{-\nu_{L}}(m L) / m L}{J_{-\nu_{R}+1}(m L)+\left(\nu_{R}-1 / 2\right) J_{-\nu_{R}}(m L) / m L}+\frac{J_{-\nu_{L}}(m L)}{J_{-\nu_{R}}(m L)}} \\
& \bar{\beta}=\frac{\frac{J_{\nu_{R}-1}(m L)-\left(\nu_{R}-1 / 2\right) J_{\nu_{R}}(m L) / m L}{J_{-\nu_{R}+1}(m L)+\left(\nu_{R}-1 / 2\right) J_{-\nu_{R}}(m L) / m L}+\frac{J_{\nu_{R}}(m L)}{J_{-\nu_{R}}(m L)}}{\frac{J_{-\nu_{L}+1}(m L)+\left(\nu_{L}-1 / 2\right) J_{-\nu_{L}}(m L) / m L}{J_{-\nu_{R}+1}(m L)+\left(\nu_{R}-1 / 2\right) J_{-\nu_{R}}(m L) / m L}+\frac{J_{-\nu_{L}}(m L)}{J_{-\nu_{R}}(m L)}} \\
& \gamma=\frac{J_{\nu_{R}-1}\left(m\left(l_{2}+L\right)\right)-\left(\nu_{R}-1 / 2\right) J_{\nu_{R}}\left(m\left(l_{2}+L\right)\right) / m\left(l_{2}+L\right)}{J_{-\nu_{R}+1}\left(m\left(l_{2}+L\right)\right)+\left(\nu_{R}-1 / 2\right) J_{-\nu_{R}}\left(m\left(l_{2}+L\right)\right) / m\left(l_{2}+L\right)} \\
& \bar{\gamma}=\frac{J_{\nu_{L}-1}\left(m\left(l_{1}+L\right)\right)-\left(\nu_{L}-1 / 2\right) J_{\nu_{L}}\left(m\left(l_{1}+L\right)\right) / m\left(l_{1}+L\right)}{J_{-\nu_{L}+1}\left(m\left(l_{1}+L\right)\right)+\left(\nu_{L}-1 / 2\right) J_{-\nu_{L}}\left(m\left(l_{1}+L\right)\right) / m\left(l_{1}+L\right)} .
\end{aligned}
$$

Eqs. (4.11) are four homogenous equations in four unknowns; for a solution to exist the coefficients (4.12) must satisfy

$$
(\gamma-\alpha)(\bar{\gamma}-\bar{\alpha})-\beta \bar{\beta}=0
$$

Combined with Eq. (4.12), the roots determine the mass eigenvalues in the spectrum.

The light bulk states which contribute to SUSY breaking satisfy $m L<<1$, $m l_{k}>1, k=1,2$. Therefore, at $z=0$ we can approximate Bessel functions as $J_{\nu}(x) \simeq(x / 2)^{\nu} / \Gamma(\nu+1)$. Eq. (4.12) becomes:

$$
\begin{aligned}
\alpha & =\mathcal{O}(1)(m L)^{2 \nu_{R}} & \beta & =\mathcal{O}(1)(m L)^{\nu_{L}+\nu_{R}} \\
\bar{\alpha} & =\mathcal{O}(1)(m L)^{2 \nu_{L}} & \bar{\beta} & =\mathcal{O}(1)(m L)^{\nu_{L}+\nu_{R}} .
\end{aligned}
$$

where $\mathcal{O}(1)$ denotes unimportant dimensionless parameters of order unity. The eigenvalue equation (4.13) simplifies to

$$
\gamma \bar{\gamma}-\mathcal{O}(1)(m L)^{2 \nu_{L}} \gamma-\mathcal{O}(1)(m L)^{2 \nu_{R}} \bar{\gamma}-\mathcal{O}(1)(m L)^{2 \nu_{L}+2 \nu_{R}}=0
$$

The information about SUSY breaking is fully encoded in the parameter $\nu_{L}$, as is evident from $(4.10)$. 
The spectrum for low $4 \mathrm{~d}$ masses contains states roughly localized on each side of the UV brane. Eqs. (4.11), (4.14) imply that the states localized on the left are characterized by $\bar{\gamma} \simeq 0, \gamma \neq 0$. Therefore in (4.15) we can ignore the last two terms on the LHS; cancelling $\gamma$ yields $\bar{\gamma}=\mathcal{O}(1)(m L)^{2 \nu_{L}}$. But since $m L<<1$, SUSY breaking from $\nu_{L}$ on the RHS is miniscule compared to SUSY breaking arising from the explicit presence of $\nu_{L}$ in the formula for $\bar{\gamma}$ in (4.12). Thus to leading order the SUSY-breaking spectrum of left-localized modes satisfies

$$
\bar{\gamma}=0 .
$$

Using $m l>>1$ near the IR branes we can use the large argument asymptotics of the Bessel functions. Since $\mathcal{O}\left(L / l_{1}\right)<<1$, (4.16) leads to

$$
\sin \left[m l_{1}-\left(\nu_{L}+1 / 2\right) \pi / 2\right]=-\frac{\nu_{L}-1 / 2}{m l} \cos \left[m l_{1}-\left(\nu_{L}+1 / 2\right) \pi / 2\right] .
$$

The supersymmetric masses take the form

$$
m=\mathcal{O}(1) \frac{n-\beta}{l_{1}},
$$

where $n$ is an integer and $\beta$ is a fraction which depends only on the supersymmetric parameters and is roughly the same for all states. This formula for the supersymmetric mass will remain true for all the bulk states in what follows. Eqs. (4.17), (4.10) give the mass splitting between the scalars and their superpartners, to leading order in $\bar{q}$ :

$$
\delta m_{L}=\mathcal{O}(1) \frac{\bar{q}^{2} L^{2}}{l_{1}}
$$

For left-localized modes, $b_{L} \sim(m L)^{2 \nu} a_{L}, b_{R} \sim(m L)^{2 \nu} a_{L}$ and $a_{R} \sim(m L)^{2 \nu} a_{L}$, so the dominant contribution to the wavefunction comes from the mode $\sim a_{L}$ localized in the region $z<0$. For $m l>>1$ the Bessel functions can be approximated by trigonometric functions for most of their support in $z$, and so the normalization condition $\int d z|\psi|^{2}=1$ requires that $l_{1} a_{L}^{2}+l_{2} a_{R}^{2}=\mathcal{O}(1)$. This yields $a_{L} \sim \mathcal{O}(1)\left(l_{1}+l_{2}(m L)^{4 \nu}\right)^{-1 / 2}$. Since $l_{1}>l_{2}$ and $m L<1$, we find $a_{L} \sim \mathcal{O}(1) l_{1}^{-1 / 2}$ to be an excellent approximation for all left-localized modes which contribute to SUSY breaking.

The overlap of the left-localized modes with the left IR brane is $\sim a_{L} \sim 1 / \sqrt{l_{1}}$, while the overlap with the right IR brane is $\sim a_{R} \sim(m L)^{\nu_{L}+\nu_{R}} / \sqrt{l_{1}} \sim(m L)^{2 \nu} / \sqrt{l_{1}}$. We renormalize the fields as in sec. 3 , to make the kinetic terms canonical. Again, the proper rescalings leading to correct couplings of canonical $4 D$ fields on the $j^{\text {th }}$-brane are: for 
bulk scalars $\Phi_{B}=\psi / a\left(l_{j}\right)^{3 / 2} \phi_{B} \sim \psi\left(l_{j} / L\right)^{3 / 2} \phi_{B}$ and for brane fields $\Phi_{b}=\phi_{4 D} / a\left(l_{j}\right) \sim$ $\phi_{4 D} l_{j} / L$. A generic interaction term on the brane is: $\left(g^{I}{ }_{J}{ }_{5 D}\right)^{2}\left(\Phi^{J}{ }_{b}\right)^{2}\left(\Phi^{I}{ }_{B}\right)^{2}$, where $I, J$ are indices denoting left $(L)$ and right $(R)$. The effective $4 D$ couplings of the left-localized bulk states $g^{L}{ }_{I} 4 D \sim g^{L}{ }_{I}$

$$
\begin{array}{ll}
g^{L}{ }_{4 D}=\frac{g^{L} L}{\sqrt{L}} & \text { to left IR brane states } \\
g^{L}{ }_{R 4 D}=\frac{g^{L}{ }_{R}(m L)^{2 \nu}}{\sqrt{L}} \sqrt{\frac{l_{2}}{l_{1}}} & \text { to right IR brane states }
\end{array}
$$

The couplings to the right IR brane states are very small, due to the high power $(m L)^{2 \nu}$, $m L<<1$. This suppression is a manifestation of tunneling.

The right-localized states are characterized by $\gamma \simeq 0, \bar{\gamma} \neq 0$. Since we are interested in the mass splitting $\delta m$ between the scalars and their superpartners to leading order in $\bar{q}$, we need to solve $\gamma=\mathcal{O}(1)(m L)^{2 \nu_{L}+2 \nu_{R}} / \bar{\gamma}=\mathcal{O}(1)(m L)^{4 \nu} \bar{q}^{2} L^{2}$. Substituting the asymptotic formula for Bessel functions into $\gamma$ in 4.12 we obtain

$\sin \left[m l_{2}-(\nu+1 / 2) \pi / 2\right]+\frac{\nu-1 / 2}{m l_{2}} \cos \left[m l_{2}-(\nu+1 / 2) \pi / 2\right]=\mathcal{O}(1)(m L)^{2 \nu}+\mathcal{O}(1)(m L)^{4 \nu} \bar{q}^{2} L^{2}$

leading to the supersymmetric masses similar to (4.18),

$$
m=\mathcal{O}(1) \frac{n-\beta}{l_{2}},
$$

and SUSY-breaking mass splitting

$$
\delta m_{R}=\mathcal{O}(1)(m L)^{4 \nu} \frac{\bar{q}^{2} L^{2}}{l_{2}}
$$

The mass splitting for the right-localized states is much smaller, by the factor $(m L)^{4 \nu}$, than for the left-localized states (4.19). This is an effect of the tunneling suppression of the wavefunction in the left throat, as seen in the relations $b_{L} \sim(m L)^{2 \nu} a_{R}, b_{R} \sim(m L)^{2 \nu} a_{R}$ and $a_{L} \sim(m L)^{2 \nu} a_{R}$. From the form of the Bessel functions for large arguments, the correct normalization of the wavefunctions requires $l_{1} a_{L}^{2}+l_{2} a_{R}^{2}=\mathcal{O}(1)$. This and $a_{L} \sim$ $a_{R}(m L)^{2 \nu}$ yields $a_{R} \sim \mathcal{O}(1)\left(l_{2}+(m L)^{4 \nu} l_{1}\right)^{-1 / 2}$. Note that since $l_{2}<l_{1}$ the two terms in the normalization formula compete. When $l_{1}>l_{2}(m L)^{-4 \nu}$ the left side contributions dominate, and hence they must not be neglected in general. Their presence indicates that when the space on the right of the barrier is tiny compared to that on the left side, it does not localize bulk modes efficiently. Those modes leak to the left. 
The overlap of the right-localized modes with the left IR brane is $\sim a_{L} \sim$ $(m L)^{2 \nu} / \sqrt{l_{2}+(m L)^{4 \nu} l_{1}}$, and with the right IR brane it is $\sim a_{R} \sim 1 / \sqrt{l_{2}+(m L)^{4 \nu} l_{1}}$. As before, the effective $4 D$ theory couplings of the right-localized bulk modes $g^{R}{ }_{i}{ }_{4 D}$ are given by

$$
\begin{array}{ll}
g^{R}{ }_{4 D}=\frac{g^{R}(m L)^{2 \nu}}{\sqrt{L}} \sqrt{\frac{l_{1}}{l_{2}+(m L)^{4 \nu} l_{1}}} & \text { to left IR brane states } \\
g_{R 4 D}^{R}=\frac{g^{R}{ }_{R}}{\sqrt{L}} \sqrt{\frac{l_{2}}{l_{2}+(m L)^{4 \nu} l_{1}}} & \text { to right IR brane states }
\end{array}
$$

The couplings to the left IR brane states are now very suppressed due to tunneling as long as $l_{1}<l_{2}(m L)^{-4 \nu}$, which is the condition for strong localization of right modes.

What about the heavy states with $m L>1$ ? For these states the barrier is lower than their (bulk) energy and therefore they can fly over it without significant suppression. Hence they are not strongly localized on different sides of the UV brane, but are only slightly asymmetric. This means that the tunneling suppression factor $\sim(m L)^{2 \nu}$ quickly converges to unity for these states, once the additional corrections in the $m L$ expansion are summed up. Hence the masses and the splittings of the left- and right-side modes become roughly the same, and are controlled by the size of the conformal box inhabited by the bulk modes, $l_{1}+l_{2}$ :

$$
\begin{aligned}
m & =\mathcal{O}(1) \frac{n-\beta}{l_{1}+l_{2}} \\
\delta m & =\mathcal{O}(1) \frac{\bar{q}^{2} L^{2}}{l_{1}+l_{2}}
\end{aligned}
$$

while the couplings are

$$
\begin{aligned}
g^{L}{ }_{L 4 D} & =\frac{g^{L} L}{\sqrt{L}} \sqrt{\frac{l_{1}}{l_{1}+l_{2}}} & g^{L}{ }_{R 4 D} & =\frac{g^{L} R}{\sqrt{L}} \sqrt{\frac{l_{2}}{l_{1}+l_{2}}} \\
g^{R}{ }_{L 4 D} & =\frac{g^{R} L}{\sqrt{L}} \sqrt{\frac{l_{1}}{l_{1}+l_{2}}} & g^{R}{ }_{R 4 D} & =\frac{g^{R} R}{\sqrt{L}} \sqrt{\frac{l_{2}}{l_{1}+l_{2}}}
\end{aligned}
$$

Here the numerator of the square root comes from the conformal rescaling by the powers on the warp factor, and is determined by the location of the brane, whereas the denominator is the normalization factor of the bulk wave function. The calculation is straightforward along the lines for the light states. This completes the construction of the bulk spectra when SUSY is broken in the entire left throat. 
It is already apparent that the left-localized modes dominate direct SUSY breaking (type 1): these modes have large mass splittings and large couplings to the left IR brane, while the right-localized modes have small mass splittings and small coulings to the left IR brane.

On the other hand, both the left- and right-localized bulk modes contribute to SUSY breaking on the right IR brane. The left-localized modes have small couplings $g^{L}{ }_{R}{ }_{4 D}$ to the right IR brane states, and the right-localized states are only weakly split. The mass splitting transmitted to the right IR brane will therefore be small We will compute the transmitted mass splittings explicitly and isolate the leading effects. But first, we determine the spectrum of bulk states if SUSY is broken only on the left IR brane.

\subsection{SUSY Breaking on the Left IR Brane}

Now we set $\bar{q}=0, q \neq 0$. In this case,

$$
\nu_{L}=\nu_{R}=\nu=\sqrt{4+m_{0}^{2} L^{2}} .
$$

The substitution of the solutions (4.6), (4.7) into the boundary conditions (4.9) with $\bar{q}=$ $0, q \neq 0$ relates the constants $a_{L, R}, b_{L, R}$ in the same way as in (4.11), except that now the explicit formulas for the coeficients in terms of Bessel functions are different. Define

$$
\hat{q}=q \frac{L}{l_{1}+L}
$$

which is well approximated by $\hat{q} \simeq q \frac{L}{l}$ for $l / L>>1$. We find

$$
\begin{aligned}
& \alpha=\bar{\alpha}=\frac{1}{2}\left[\frac{J_{\nu-1}(m L)-(\nu-1 / 2) J_{\nu}(m L) / m L}{J_{-\nu+1}(m L)+(\nu-1 / 2) J_{-\nu}(m L) / m L}-\frac{J_{\nu}(m L)}{J_{-\nu}(m L)}\right] \\
& \beta=\bar{\beta}=\frac{1}{2}\left[\frac{J_{\nu-1}(m L)-(\nu-1 / 2) J_{\nu}(m L) / m L}{J_{-\nu+1}(m L)+(\nu-1 / 2) J_{-\nu}(m L) / m L}+\frac{J_{\nu}(m L)}{J_{-\nu}(m L)}\right] \\
& \gamma=\frac{J_{\nu-1}\left(m\left(l_{2}+L\right)\right)-(\nu-1 / 2) J_{\nu}\left(m\left(l_{2}+L\right)\right) / m\left(l_{2}+L\right)}{J_{-\nu+1}\left(m\left(l_{2}+L\right)\right)+(\nu-1 / 2) J_{-\nu}\left(m\left(l_{2}+L\right)\right) / m\left(l_{2}+L\right)} \\
& \bar{\gamma}=\frac{J_{\nu-1}\left(m\left(l_{1}+L\right)\right)-(\nu-1 / 2) J_{\nu}\left(m\left(l_{1}+L\right)\right) / m\left(l_{1}+L\right)+\hat{q} J_{\nu}\left(m\left(l_{1}+L\right)\right) / m}{J_{-\nu+1}\left(m\left(l_{1}+L\right)\right)+(\nu-1 / 2) J_{-\nu}\left(m\left(l_{1}+L\right)\right) / m\left(l_{1}+L\right)-\hat{q} J_{-\nu}\left(m\left(l_{1}+L\right)\right) / m}
\end{aligned}
$$

The coefficients must still satisfy a consistency condition, leading to the eigenvalue equation (4.13) as before. 
For light states $m L<<1, m l_{k}>1$ we again approximate the Bessel functions at the IR and UV branes by their asymptotic values for large and small arguments respectively. As before,

$$
\alpha=\mathcal{O}(1)(m L)^{2 \nu} \quad \beta=\mathcal{O}(1)(m L)^{2 \nu}
$$

and therefore the eigenvalue equation becomes

$$
\gamma \bar{\gamma}-\mathcal{O}(1)(m L)^{2 \nu}(\gamma+\bar{\gamma})-\mathcal{O}(1)(m L)^{4 \nu}=0
$$

Now the SUSY breaking scale is completely contained within $\bar{\gamma}$.

The bulk spectrum again naturally splits into left- and right-localized states. The left-localized states again have $b_{L} \sim(m L)^{2 \nu} a_{L}, b_{R} \sim(m L)^{2 \nu} a_{L}, a_{R} \sim(m L)^{2 \nu} a_{L}$, and $\bar{\gamma} \simeq(m L)^{2 \nu}<<\gamma \neq 0$. Because we wish to detemine the mass splitting $\delta m$ to leading order in $\hat{q}$, it is sufficient to consider $\bar{\gamma}=0$, or

$$
J_{\nu-1}\left(m l_{1}\right)=\left(\frac{\nu-1 / 2}{m l}-\frac{\hat{q}}{m}\right) J_{\nu}\left(m l_{1}\right)
$$

which at the IR brane can be approximated by:

$$
\sin \left[m l_{1}-(\nu+1 / 2) \pi / 2\right]=\left(\frac{\hat{q}}{m}-\frac{\nu-1 / 2}{m l_{1}}\right) \cos \left[m l_{1}-(\nu+1 / 2) \pi / 2\right]
$$

The supersymmetric masses are the solutions of this equation when $q=0$, and are given by Eq. (4.18). We will find below that $q$ is within an order of magnitude or two of $1 / L$. Since $\hat{q} l_{1} \simeq q L$, the ratio $\hat{q} / m<1$ for some of the relevant states, and $\hat{q} / m>1$ for the rest. These cases must be treated separately in Eq. (4.33). For the heavy states, the SUSY breaking terms are small perturbations, so that $\delta m \sim \hat{q} /\left(m l_{1}\right)$. For the light states, the SUSY breaking terms are a large perturbation, leading to a change in the mass of order of $\mathcal{O}(1) / l_{1}$. The results for the mass splittings are

$$
\begin{array}{rlrl}
\delta m_{L} & =\mathcal{O}(1) \frac{1}{l_{1}} & & \text { when } m<\hat{q} \\
\delta m_{L}=\mathcal{O}(1) \frac{\hat{q}}{l_{1} m} & & \text { when } m>\hat{q}
\end{array}
$$

The effective four-dimensional couplings are the same as in type A) breaking as summarized in Eq. (4.20).

The masses, splittings, and couplings of the right-localized states are computed in the same way. For these states, $b_{L} \sim(m L)^{2 \nu} a_{R}, b_{R} \sim(m L)^{2 \nu} a_{R}, a_{L} \sim(m L)^{2 \nu} a_{R}, \gamma \simeq 0$, and 
$\bar{\gamma} \neq 0$. Determining the mass splitting is subtler than before. As $l_{2}>>L$, the eigenvalue equation is approximately:

$$
\begin{aligned}
J_{\nu-1}\left(m l_{2}\right) & -\frac{\nu-1 / 2}{m l_{2}} J_{\nu}\left(m l_{2}\right)=\mathcal{O}(1)(m L)^{2 \nu} \\
& +\mathcal{O}(1)(m L)^{4 \nu} \frac{J_{-\nu+1}\left(m l_{1}\right)+(\nu-1 / 2) J_{-\nu}\left(m l_{1}\right) / m l_{1}-\hat{q} J_{-\nu}\left(m l_{1}\right) / m}{J_{\nu-1}\left(m l_{1}\right)-(\nu-1 / 2) J_{\nu}\left(m l_{1}\right) / m l_{1}+\hat{q} J_{\nu}\left(m l_{1}\right) / m} .
\end{aligned}
$$

When $q=0$ the roots yield the masses of the light supersymmetric modes, which are of the same form as (4.22). Solving perturbatively for $\delta m$ as a function of $\hat{q}$, we find that for $\hat{q}>>m$

$$
J_{\nu-1}\left(m l_{2}\right)-\frac{\nu-1 / 2}{m l_{2}} J_{\nu}\left(m l_{2}\right)=\mathcal{O}(1)(m L)^{2 \nu}+\mathcal{O}(1)(m L)^{4 \nu},
$$

where the first term on the RHS is a supersymmetric shift of the mass $\sim(m L)^{2 \nu} / l_{2}$ which merely renormalizes $\beta$ in (4.18), while the second term arises because of the SUSY breaking. Hence the mass splitting induced by the SUSY breaking terms is $\delta m \sim(m L)^{4 \nu} / l_{2}$ when $\hat{q} / m>1$, again using the large argument asymptotics of Bessel functions. When $\hat{q} / m<1$, we find

$$
J_{\nu-1}\left(m l_{2}\right)-\frac{\nu-1 / 2}{m l_{2}} J_{\nu}\left(m l_{2}\right)=\mathcal{O}(1)(m L)^{2 \nu}+\mathcal{O}(1)(m L)^{4 \nu}+\mathcal{O}(1)(m L)^{4 \nu} \frac{\hat{q}}{m}
$$

and so the SUSY breaking terms induce mass splitting $\delta m \sim(m L)^{4 \nu} \hat{q} /(m l)$. (Note that the second term on the RHS of ((4.37)) does not arise from SUSY breaking, unlike the similar term in Eq. ((4.36) ).) Summarizing, we find that

$$
\begin{aligned}
\delta m_{R}=\mathcal{O}(1)(m L)^{4 \nu} \frac{1}{l_{2}} & \text { when } m<\hat{q} \\
\delta m_{R}=\mathcal{O}(1)(m L)^{4 \nu} \frac{\hat{q}}{l_{2} m} & \text { when } m>\hat{q}
\end{aligned}
$$

In this case the correct normalization of the wavefunctions is $a_{R}=\mathcal{O}(1)\left(l_{2}+\right.$ $\left.(m L)^{4 \nu} l_{1}\right)^{-1 / 2}$. Again for $l_{1}>l_{2}(m L)^{-4 \nu}$ the left side contributions would dominate, indicating that the modes with such a high mass cease to be localized on the right. The effective four-dimensional couplings of the right-localized modes are identical to that in type A) SUSY breaking, as seen in Eq. ((4.24)).

The couplings of the heavy states with $m L>1$ behave qualitatively the same as when SUSY is broken in the whole left throat. The barrier is too low compared to their bulk energy to lead to significant localization on one side or the other. The tunneling 
suppression factor $\sim(m L)^{2 \nu}$ is replaced by a function which quickly converges to unity when $m L>>1$. Hence the couplings rapidly converge to

$$
\begin{array}{ll}
g^{L}{ }_{L 4 D}=\frac{g^{L} L}{\sqrt{L}} \sqrt{\frac{l_{1}}{l_{1}+l_{2}}} & g^{L}{ }_{R 4 D}=\frac{g^{L} R}{\sqrt{L}} \sqrt{\frac{l_{2}}{l_{1}+l_{2}}} \\
g^{R}{ }_{L 4 D}=\frac{g^{R} L}{\sqrt{L}} \sqrt{\frac{l_{1}}{l_{1}+l_{2}}} & g^{R}{ }_{R 4 D}=\frac{g^{R}}{\sqrt{L}} \sqrt{\frac{l_{2}}{l_{1}+l_{2}}}
\end{array}
$$

The masses are controlled by the size of the conformal box inhabited by the bulk modes, $l_{1}+l_{2}$ :

$$
m=\mathcal{O}(1) \frac{n-\beta}{l_{1}+l_{2}}
$$

as are the mass splittings, which however also depend inversely on the mass of the state, as dictated by the SUSY-breaking boundary condition

$$
\delta m=\mathcal{O}(1) \frac{\hat{q}}{m\left(l_{1}+l_{2}\right)} .
$$

This completes the determination of the spectra of bulk modes.

\subsection{Transmission to brane worldvolume fields}

Given the spectrum of the bulk modes and their couplings to the IR branes, we can compute the induced mass splitting of scalar superpartners of $4 D$ fermions. The most interesting case is case 2) where the splitting is suppressed by tunneling effects. We will compute the splitting for types A) and B) SUSY breaking. We will also discuss case 1) in order to isolate tunneling effects in case 2).

Suppose there are brane-localized scalars and fermions $\phi^{L, R_{b}}$ and $\lambda^{L, R}{ }_{b}$, and bulk scalars and fermions $\phi^{L, R}{ }_{B}$ and $\lambda^{L, R}{ }_{B}$. Indices $L, R$ refer to the left- and right-localized states, both in the throat and on the IR brane. Assume the $4 \mathrm{~d}$ effective Lagrangian contains generic quartic couplings between brane and bulk KK scalars, and Yukawa couplings between brane and bulk scalars and fermions, of the form

$$
\mathcal{L}=\cdots+\left(g^{I}{ }_{J 5 D}\right)^{2}\left(\phi_{b}^{J}\right)^{2}\left(\phi_{B}^{I}\right)^{2}+g^{I}{ }_{5 D} \phi_{b}^{J} \lambda_{b}{ }_{b} \lambda^{I}{ }_{B}+\cdots
$$

Since $\left[\phi_{B}\right]=3 / 2$ is the canonical dimension of a $5 \mathrm{~d}$ scalar field, one expects

$$
g_{J 5 D}^{I} \sim \frac{1}{\sqrt{M_{5}}} .
$$


Renormalizing the fields to have canonical $4 D$ kinetic terms, we find that the induced $4 D$ dimensionless couplings are $g^{I}{ }_{J}{ }_{4 D} \sim \frac{1}{\sqrt{M_{5} L}}$. Our results for both type A) and type B) SUSY breaking can be summarized as follows, recalling that $l_{1}>l_{2}$ :

$$
\begin{gathered}
g_{L 4 D}^{L}=\frac{\mathcal{O}(1)}{\sqrt{M_{5} L}} \quad g_{R 4 D}^{R}=\frac{\mathcal{O}(1)}{\sqrt{M_{5} L}} \sqrt{\frac{l_{2}}{l_{2}+f^{2}(m L) l_{1}}} \\
g_{{ }_{J}{ }_{4 D}}=\frac{\mathcal{O}(1)}{\sqrt{M_{5} L}} f(m L) \sqrt{\frac{l_{J}}{l_{I}+f^{2}(m L) l_{J}}}(I \neq J) .
\end{gathered}
$$

Here $f$ is the square root of the transmission coefficient through the barrier, which has the asymptotic forms:

$$
f \simeq\left\{\begin{array}{ll}
(m L)^{2 \nu} & m L<<1 \\
1 & m L>>1
\end{array} .\right.
$$

The "off-diagonal" couplings are always much smaller than the "diagonal" ones because of the tunneling.

The masses of the bulk particles are

$$
m(B, F)_{L, R}=\alpha_{L, R}(n) \frac{n+\beta(B, F, n)_{L, R}}{l_{1,2}+h(n) l_{2,1}}
$$

where the coefficients $\alpha$ and $\beta$ depend softly on $M_{0}, n$. The difference in $\beta$ between bosons and fermions parameterizes SUSY breaking. The function $h$ encodes the behavior of masses and splittings in the limit when tunneling suppression vanishes. It vanishes near zero and asymptotes to unity for large argument, to allow for the transition between, e.g., (4.18), (4.19), (4.22), (4.23) to (4.25). Its precise form is not important for our conclusions. Note that for the left-localized modes, we can completely ignore the term with $h$ since we assume $l_{1}>l_{2}$.

Assume that the fermions living on the IR brane are protected from mass shifts, e.g. because they belong in chiral gauge representations. The scalars are not. A naive estimate of the mass shift of the scalars on the IR brane would run as follows. The contribution from one-loop diagrams involving bulk fields $\phi^{I}{ }_{B}, \lambda^{I}{ }_{B}$ can be computed state-by-state, by first performing the loop integrals over 4-momenta, and then subtracting fermionic from bosonic contributions. This gives approximately

$$
\left(\delta m^{J}{ }_{I b}\right)^{2} \sim\left(g^{I}{ }_{J} 4 D\right)^{2}\left[m^{2}(B)-m^{2}(F)\right] \sim\left(g^{I}{ }_{J}{ }_{4 D}\right)^{2} m_{I} \delta m_{I}
$$

for each KK state in the loop, where $m_{I}$ and $\delta m_{I}$ are the bulk supersymmetric mass and the boson-fermion bulk mass splitting induced by SUSY breaking, respectively. If we 
insert the appropriate bulk couplings and splittings and sum over the entire tower of bulk KK modes, we get a divergent answer, $\left(\delta m_{I b}^{J}\right)^{2}=\sum_{m \sim 1 / l}^{\Lambda_{U V}}\left(g_{J}^{I}{ }_{4 D}\right)^{2} m_{I} \delta m_{I}$, suggesting a cutoff $\Lambda_{U V}$ a priori distinct from the UV cutoff used to compute the state-by-state mass splittings.

This divergence is artificial. In the 5D picture of the calculation the SUSY breaking transmission is generated by Feynman diagrams with bulk particles in the loops and wall particles in the external legs. The summation over the $4 D \mathrm{KK}$ states is the "integration" over the momentum in the fifth direction in the higher-dimensional theory. In the coordinate representation this calculation is perfectly well-defined unless the external particles are at coincident points. This issue is handled in the standard way by renormalization.

In the momentum representation $p_{5}$ is discretized due to the compactness of our background in the $z$ direction. By first doing the integrals over the $4 D$ momenta, we would then sum over divergent quantities, requiring independent regularization for both the sum and the integrals. This is not the correct way to proceed. Instead, we use the fact that field theory regulates itself away from coincident points in coordinate representation, sum over $p_{5}$ first, and then integrate over the 4-momenta and renormalize the final answer, if need be. Since the loop propagators depend inversely on the square of KK mass, this sum converges. Our method is very similar to the regularization techniques employed in [35], [36], [32].

Now before doing any momentum sums or integrations, the mass splitting for scalars on the IR brane is:

$$
\left(\delta m_{I b}^{J}\right)^{2}=\frac{2}{(2 \pi)^{4}} \sum_{m}\left(g_{J}^{I}{ }_{4 D}\right)^{2}\left(\int \frac{d^{4} p}{p^{2}+m^{2}(B)}-\int \frac{d^{4} p}{p^{2}+m^{2}(F)}\right)
$$

Here the factor of $2 /(2 \pi)^{4}$ comes from the symmetry factor for the Lagrangian (4.42) and the usual normalization for loop integrals. The boson and fermion masses are $m(B, F)=$ $\alpha\left(n+\beta_{B, F}\right) / l$. The couplings and the parameters $\beta_{B, F}, l$ explicitly depend on the masses as explained in (4.46).

The induced mass splittings will generally be differences of sums of the form

$$
\left.S_{I}=\frac{\mathcal{O}(1)}{8 \pi^{4} M_{5} L} \sum_{n} F\left[\frac{\alpha\left(n+\beta_{I}\right) L}{l}\right)\right] \int \frac{d^{4} p}{p^{2}+\alpha^{2}\left(n+\beta_{I}\right)^{2} / l^{2}} .
$$

Here $F=g^{2} M_{5} L$ and its form can be readily read from (4.44), and $l$ is the denominator in (4.46). A crucial property of $F$ is that it converges to a constant for large arguments. 
Indeed, on the real axis $F<l_{1} / l_{2}$ for any of the couplings (4.44). We can now use a standard technique for the summation of infinite series. Consider the contour integral

$$
\int_{C} d z F\left(\frac{\alpha L z}{l}\right) \frac{\cot \left[\pi\left(z-\beta_{I}\right)\right]}{p^{2}+\alpha^{2} z^{2} / l^{2}}
$$

where the contour consists of a large circle oriented counterclockwise and small clockwise circles around all the poles of the integrand inside the large circle. Then take the limit where the radius of the big circle goes to infinity, such that all the poles are enclosed by it. Since $F$ goes to a constant for large arguments, the integral along the large circle vanishes because of the denominator in (4.50). Therefore the integrals along the small circles add up to zero. They can be readily computed by the residue theorem. The dependence of the parameters $\beta$ on the mass is very soft, and we can ignore their effect on deforming the poles of the cotangent away from the integers.

We also need to account for the poles in $F$, which are required because $F$ is not constant and is bounded for large values of the argument. By continuity, any such poles in $F$ are located outside the circle of radius $1 / L$. They could be imaginary, in which case they would correspond to a state with a large negative (mass) ${ }^{2}$. However, it is very straightforward to understand such poles: the states heavy enough to cross the barrier classically are not well-localized, and so the left and the right states mesh together strongly. If one naively ignores the presence of the states from one side of the barrier, one is "reminded" of them precisely by these poles. Hence, such imaginary poles would just correspond to states localized on the other side of the barrier, which are added in in the other sum. They should not be included in the tunneling calculation twice. If the poles have a large real part, this means that the the approximate eigenvalue equations which we have used to find the spectrum receive corrections, as can be seen from the form of the functions (4.12) or (4.29). Both the transmission coefficient and the eigenvalue equation depend on those parameters, and after the heavy masses are appropriately corrected, these poles are accounted for in the improved summation over the states in the KK towers. Therefore we conclude that any such poles of $F$ are spurious and should be circumnavigated by Wick rotation, which we have already performed. Hence they will not contribute to the momentum integrals, and we can ignore them. With our approximations, the only poles we need to keep are at the integers along the real axis, and at the complex points $z= \pm i l p / \alpha$. Therefore:

$$
\begin{aligned}
\sum_{n} \frac{F\left(\alpha\left(n+\beta_{I}\right) L / l\right)}{p^{2}+\alpha^{2}\left(n+\beta_{I}\right)^{2} / l^{2}} & =-\frac{l F(i p L)}{2 i \alpha p} \cot \left[\pi\left(\frac{i l p}{\alpha}-\beta_{I}(i p L)\right)\right] \\
& -\frac{l F(-i p L)}{2 i \alpha p} \cot \left[\pi\left(\frac{i l p}{\alpha}+\beta_{I}(-i p L)\right)\right]
\end{aligned}
$$


Since $F(x)$ is real on the real axis, the RHS is obviously real.

Now we isolate the terms in (4.51) which give the UV divergence in (4.49). Since

$$
\cot \left[\pi\left(\frac{i l p}{\alpha} \pm \beta_{I}\right)\right]=-i-i \frac{2}{e^{2 \pi l p / \alpha \mp 2 i \pi \beta_{I}}-1}
$$

we can write $S_{I}$ as

$$
\begin{aligned}
S_{I}= & \frac{\mathcal{O}(1)}{8 \pi^{4} M_{5} L} \frac{l}{2 \alpha} \int \frac{d^{4} p}{p}(F(i p L)+F(-i p L)) \\
& +\frac{\mathcal{O}(1)}{8 \pi^{4} M_{5} L} \frac{l}{2 \alpha} \int \frac{d^{4} p}{p}\left(\frac{2 F(i p L)}{e^{2 \pi l p / \alpha-2 i \pi \beta_{I}(i p L)}-1}+\frac{2 F(-i p L)}{e^{2 \pi l p / \alpha+2 i \pi \beta_{I}(-i p L)}-1}\right)
\end{aligned}
$$

The first integral in (4.52) is divergent. However it is independent of the particle quantum numbers, and so cancels out of (4.48). We will however keep this term in mind, because it will give the dominant, UV-finite, contribution to the gaugino mass (see §4.5). The second integral is finite, and does not cancel out of (4.48) because of bulk SUSY breaking. After the angular integration, the remaining integral is

$$
S_{I}^{\text {finite }}=\frac{\mathcal{O}(1) l}{4 \pi^{2} \alpha M_{5} L} \int_{0}^{\infty} d p p^{2}\left(\frac{F(i p L)}{e^{2 \pi l p / \alpha-2 i \pi \beta_{I}(i p L)}-1}+\frac{F(-i p L)}{e^{2 \pi l p / \alpha+2 i \pi \beta_{I}(-i p L)}-1}\right)
$$

and it is the leading contribution to (4.48). This integral is obviously finite due to the exponential momentum cutoff in the denominator, and is well behaved in the IR due to the measure factor. Because of the cutoff the integral is dominated by the contributions $p \sim 1 / l$. We can evaluate it approximately by the saddle point method, once we have the explicit form of $F$. Thus the states which determine the mass splittings of the wall chiral multiplets are the light bulk states. The heavy states would make it more easily over the barrier, but decoupling reduces their effect on the transmission. We can now compute the wall chiral multiplet mass splittings case-by-case.

\section{Direct SUSY Breaking}

For direct SUSY breaking - case 1) - we have $F=1, \beta_{B, F}=$ const and $l=l_{1}$ (4.44), (4.46). Substituting (4.54) into (4.48), using $\beta_{B}-\beta_{F}=\bar{q}^{2} L^{2}$ (4.19), we evaluate the integrals by the saddle point method. We find, using $\sin \left(2 \pi\left|\beta_{B}\right|\right) \sim \mathcal{O}(1)$,

$$
\left(\delta m^{L}{ }_{L b}\right)^{2} \sim \mathcal{O}(1) \frac{\bar{q}^{2} L}{M_{5}} \frac{1}{l_{1}^{2}} .
$$

Due to decoupling, only the lightest modes contribute significantly to supersymmetry breaking induced on the left IR brane, even though couplings are independent of the 
KK masses. Obviously the mass splittings induced on the brane are given by the simple exponential scaling induced by the conformal factor on the brane. This is similar to [37].

\section{Tunneling mediation with left bulk SUSY breaking}

Let us turn to tunneling mediation - case 2) - starting with type A) SUSY breaking. We compute the mass splittings on the right IR brane as induced by loops of the bulk states. For left-localized states, $F=x^{4 \nu} l_{2} / l_{1}, \beta_{B}-\beta_{F}=\bar{q}^{2} L^{2}$ and $l=l_{1}$ (4.19),(4.44). Therefore

$$
\left(\delta m^{R}{ }_{L b}\right)^{2} \sim \mathcal{O}(1) \frac{\bar{q}^{2} L}{M_{5}} \frac{l_{2}}{l_{1}^{3}}\left(\frac{L}{l_{1}}\right)^{4 \nu} .
$$

Compared to the direct SUSY breaking (4.55), there is additional dependence on the ratio $l_{2} / l_{1}$. This is precisely the rescaling of dimensionful parameters in the AdS space. The right IR brane resides closer to the UV brane, and the couplings on it are smaller than those on the left IR brane precisely by this ratio. In addition, there is a significant

suppression factor $\left(\frac{L}{l_{1}}\right)^{4 \nu}$, over and above the splitting in direct transmission (4.55). This is an exponential suppression of the scale of SUSY breaking due to tunneling.

Similarly, for the right-localized bulk states, $F=l_{2} /\left(l_{2}+x^{4 \nu} l_{1}\right), \beta_{B}-\beta_{F}=\bar{q}^{2} L^{2} x^{4 \nu}$ and $l=l_{2}$ (4.23),(4.44). The mass splitting is:

$$
\left(\delta m^{R}{ }_{R b}\right)^{2} \sim \mathcal{O}(1) \frac{\bar{q}^{2} L}{M_{5}} \frac{1}{l_{2}\left(l_{2}+\left(L / l_{2}\right)^{4 \nu} l_{1}\right)}\left(\frac{L}{l_{2}}\right)^{4 \nu} .
$$

There are two extreme limits, depending on which term dominates the denominator. In these limits, the mass splitting transmitted to the right IR brane by the right-localized bulk multiplets is

$$
\left(\delta m_{S M}\right)^{2} \sim \mathcal{O}(1) \frac{\bar{q}^{2} L}{M_{5}} \times \begin{cases}\frac{1}{l_{2}^{2}}\left(\frac{L}{l_{2}}\right)^{4 \nu} & l_{1}<l_{2}\left(\frac{l_{2}}{L}\right)^{4 \nu} \\ \frac{1}{l_{2} l_{1}} & l_{1}>l_{2}\left(\frac{l_{2}}{L}\right)^{4 \nu} .\end{cases}
$$

The first case is again suppressed relative to (4.55) by the factor of $(L / l)^{4 \nu}$. Comparing eqs. (4.56) and (4.58) for the relevant values of parameters we find that the right-localized states always give a dominant contribution to the transmission of SUSY breaking to the SM fields on the right IR brane as long as $l_{1}>l_{2}$. 


\section{Tunneling mediation with left brane-localized SUSY breaking}

Finally, we repeat these calculations for type B) SUSY breaking. For the contribution of the left-localized states, $F=x^{4 \nu} l_{2} / l_{1}, \beta_{B}-\beta_{F}=q L^{2} /\left(l_{1} x\right)$ and $l=l_{1}$ in (4.34), (4.44). This gives

$$
\left(\delta m^{R}{ }_{L b}\right)^{2} \sim \mathcal{O}(1) \frac{q}{M_{5}} \frac{l_{2}}{l_{1}^{3}}\left(\frac{L}{l_{1}}\right)^{4 \nu} .
$$

Again, the factor of $\left(\frac{L}{l_{1}}\right)^{4 \nu}$ reflects tunneling, while the power $l_{2} / l_{1}$ represents the usual coupling rescaling.

For the right-localized states, $F=l_{2} /\left(l_{2}+x^{4 \nu} l_{1}\right), \beta_{B}-\beta_{F}=x^{4 \nu} q L^{2} /\left(l_{1} x\right)$ and $l=l_{2}$ in $(4.38),(4.44)$, and therefore

$$
\left(\delta m_{R b}^{R}\right)^{2} \sim \mathcal{O}(1) \frac{q}{M_{5}} \frac{1}{l_{2}\left(l_{2}+\left(L / l_{2}\right)^{4 \nu} l_{1}\right)}\left(\frac{L}{l_{2}}\right)^{4 \nu} .
$$

As for the transmission of left bulk SUSY breaking, we again focus on two extreme cases, $l_{1}<l_{2}\left(\frac{l_{2}}{L}\right)^{4 \nu}$ and $l_{1}>l_{2}\left(\frac{l_{2}}{L}\right)^{4 \nu}$. In either case, we can see that the right-localized modes give dominant contribution to the splittings of the right brane multiplets. The transmitted mass splittings on the right IR brane from the SUSY breaking which occurs on the left IR brane is

$$
\left(\delta m_{S M}\right)^{2} \sim \mathcal{O}(1) \frac{q}{M_{5}} \times \begin{cases}\frac{1}{l_{2}^{2}}\left(\frac{L}{l_{2}}\right)^{4 \nu} & l_{1}<\left(\frac{l_{2}}{L}\right)^{4 \nu} l_{2} \\ \frac{1}{l_{1} l_{2}} & l_{1}>\left(\frac{l_{2}}{L}\right)^{4 \nu} l_{2}\end{cases}
$$

The main contribution to $\delta m_{S M}$ is induced by the modes living on the right side. Just like (4.58), $\delta m_{S M}$ is dramatically suppressed compared to case 1 ). The calculations verify the simple intuition that, to transmit the SUSY breaking from the left throat to the right throat, the modes pay an exponential price to tunnel through the potential barrier at the UV brane.

\subsection{R-symmetry Breaking and Gaugino Masses}

In the previous sections we have assumed that SUSY breaking is mediated by the bulk scalars, while bulk fermions were protected from the breaking effects. However, in order to generate gaugino masses, bulk fermions must also couple to SUSY-breaking physics. Only through their exchange is it possible to break R-symmetry, which is necessary for gaugino mass generation.

We will consider in detail a scenario where SUSY breaking is confined to the left IR brane. One could, in principle, break SUSY in the entire left throat. However, the 
combination of the Dirac mass term (needed to avoid tachyons among the scalar superpartners) and the Majorana mass term (necessary to break R-symmetry and generate gaugino masses) for bulk fermions complicates the calculation considerably.

Our model consists of two chiral bulk fermions, $\zeta$ (undotted, two-component spinor c.f. [38] for notation) and $\chi$ (dotted spinor) minimally coupled to gravity and coupled to each other by a bulk Dirac mass $M$, and left-brane-localized Majorana terms induced by SUSY breaking. In this section we will work with the metric signature $(+----)$.

A simple action for the fermions is:

$$
\begin{aligned}
S_{F} & =\int d^{5} x \sqrt{g_{5}}\left(\frac{i}{2} \bar{\Psi} \Gamma^{A} \overleftrightarrow{\partial}_{A} \Psi+\frac{1}{8} \omega_{b c . A} \bar{\Psi}\left\{\Gamma^{A}, \sigma^{b c}\right\} \Psi-M \bar{\Psi} \Psi\right) \\
& +\int d^{4} x \sqrt{g_{4}}\left(q_{1}\left[\zeta^{T} i \sigma^{2} \zeta-\zeta^{\dagger} i \sigma^{2} \zeta^{*}\right]-q_{2}\left[\chi^{T} i \sigma^{2} \chi-\chi^{\dagger} i \sigma^{2} \chi^{*}\right]\right)
\end{aligned}
$$

Here $\Psi^{T}=(\zeta, \chi)$, and

$$
A \overleftrightarrow{\partial} B=A \partial B-(\partial A) B
$$

Also, $\gamma^{A}=\left(\gamma^{\mu}, \operatorname{diag}(-i, i)\right)$ are $5 D$ Dirac matrices, $\Gamma^{A}=E_{B}{ }^{A} \gamma^{B}$, and $\sigma^{a b}=\frac{i}{2}\left[\gamma^{a}, \gamma^{b}\right]$. $g_{A B}^{5 D}=\eta_{C D} e^{C}{ }_{A} e^{D}{ }_{B}$ defines both the funfbein $e^{A}{ }_{B}$ and its inverse $E_{B}{ }^{A}, e^{A}{ }_{B} E_{C}{ }^{B}=\delta^{A}{ }_{B}$. Hermitean conjugation is denoted by $\dagger$, complex conjugation by $*$ and transposition by $T$. Dirac conjugates are defined on the tangent space $\bar{\Psi}=\Psi^{\dagger} \gamma^{0}$. Finally, will will use the spinor representation of the Dirac matrices:

$$
\gamma^{\mu}=\left(\begin{array}{cc}
0 & \sigma^{\mu} \\
\bar{\sigma}^{\mu} & 0
\end{array}\right)
$$

and $\sigma^{\mu}=\operatorname{diag}\left(1, \sigma^{k}\right), \bar{\sigma}^{\mu}=\operatorname{diag}\left(1,-\sigma^{k}\right)$ and $\sigma^{k}$ are the usual Pauli matrices.

Before proceeding we note that because the Majorana terms live on the left IR brane, they don't need to be the symplectic Majorana masses characteristic for bulk $5 D$ fermions; our background breaks invariance under the AdS isometry group. We will see below that one of the mass parameters $q_{i}$ must vanish identically. Furthermore, because the $5 D$ fermions dimension 2, the coefficients $q_{1}, q_{2}$ of the Majorana terms on the left IR brane are dimensionless. This effects the dependence of the induced mass splitting on the exponentials that emerge from the width of the barrier between the throats, leading to different SUSY-breaking behavior than that discussed previously for bosons. The $4 D$ scale which emerges in the effective $4 D$ description of these modes is induced by the normalization of the modes and their overlap with the left IR brane, and can therefore be small. 
We emphasize that while the IR branes are endowed with orbifold boundary conditions, the UV brane is not. For fermions this affects physics in a crucial way. An immediate consequence is that the bulk Dirac mass parameter $M$ is continuous across the UV brane, $M(-z)=M(z)$. As a result the bulk parity operator acting on fermions is not the same on all the branes. We will see below that parity inversion about the UV brane interchanges the chiral spinors while it does not around IR orbifolds. If we take the realization of parity on the UV brane as "natural", then the choice of parity assignments on different IR branes can lead to twisted bulk fermions, breaking left-right symmetry about the UV brane. Such bulk fermions are not degenerate even in the supersymmetric limit, despite the apparent symmetry of (2.1). We also note that these boundary conditions on the UV brane would be compatible with additional UV brane-localized supersymmetric fermion mass terms, analogous to the $\sim \delta$-function supersymmetric terms in (4.3) which we have ignored. We will ignore such terms here too.

Note that the bosons of $\S 4.3$ and fermions treated here are not supersymmetric partners of each other. The correct sfermions would have to satisfy different boundary conditions from those we have chosen in $\S 4.3$. We will ignore a detailed treatment of the latter here and merely point out the relations later on. Our analysis is precise for the case when SUSY breaking on the left IR brane is completely confined to the fermion sector.

Now we wish to find the equations of motion for the fermions. Because the background (2.1) is conformally flat, $\omega_{b c . A} \bar{\Psi}\left\{\Gamma^{A}, \sigma^{b c}\right\} \Psi=0$ identically. Substituting the ansatz (2.1) into (4.62), after straightforward algebra we find

$$
\begin{aligned}
S_{F} & =\int d^{4} x d z\left\{a^{4}\left[\chi^{\dagger} i \sigma^{\mu} \partial_{\mu} \chi+\zeta^{\dagger} i \bar{\sigma}^{\mu} \partial_{\mu} \zeta+\chi^{\dagger}\left(\frac{1}{2} \overleftrightarrow{\partial}_{z} \zeta+2 \frac{a^{\prime}}{a} \zeta\right)-\zeta^{\dagger}\left(\frac{1}{2} \overleftrightarrow{\partial}_{z} \chi+2 \frac{a^{\prime}}{a} \chi\right)\right]\right. \\
& \left.-M a^{5}\left(\chi^{\dagger} \zeta+\zeta^{\dagger} \chi\right)+\delta\left(z+l_{1}\right) a^{4}\left(q_{1}\left[\zeta^{T} i \sigma^{2} \zeta-\zeta^{\dagger} i \sigma^{2} \zeta^{*}\right]-q_{2}\left[\chi^{T} i \sigma^{2} \chi-\chi^{\dagger} i \sigma^{2} \chi^{*}\right]\right)\right\} .
\end{aligned}
$$

Defining the effective $4 D$ theory fermions by the change of variables $Z=a^{2} \zeta, X=a^{2} \chi$ removes the cross terms $\sim a^{\prime} \Psi^{\prime}$ from the action, so that:

$$
\begin{aligned}
S_{F}=\int d^{4} x d z & \left\{X^{\dagger} i \sigma^{\mu} \partial_{\mu} X+Z^{\dagger} i \bar{\sigma}^{\mu} \partial_{\mu} Z+\frac{1}{2} X^{\dagger} \overleftrightarrow{\partial_{z}} Z-\frac{1}{2} Z^{\dagger} \overleftrightarrow{\partial_{z}^{X}}-M a\left(X^{\dagger} Z+Z^{\dagger} X\right)\right. \\
& \left.+\delta\left(z+l_{1}\right)\left(q_{1}\left[Z^{T} i \sigma^{2} Z-Z^{\dagger} i \sigma^{2} Z^{*}\right]-q_{2}\left[X^{T} i \sigma^{2} X-X^{\dagger} i \sigma^{2} X^{*}\right]\right)\right\}
\end{aligned}
$$

The kinetic terms are now independent of the warp factor, which only enters through the bulk Dirac mass term. This reflects the conformal symmetry of a massless fermion; the symmetry is broken only by the mass terms. 
The fermion equations of motion are:

$$
\begin{aligned}
& i \bar{\sigma}^{\mu} \partial_{\mu} Z-X^{\prime}=M a X-2 q_{2} \delta\left(z+l_{1}\right) i \sigma^{2} Z^{*} \\
& i \sigma^{\mu} \partial_{\mu} X+Z^{\prime}=M a Z+2 q_{1} \delta\left(z+l_{1}\right) i \sigma^{2} X^{*} .
\end{aligned}
$$

These equations can be cast in 4-component form by introducing

$$
\Sigma=\left(\begin{array}{c}
Z \\
X
\end{array}\right) \quad D=\left(\begin{array}{cc}
\partial_{z} & i \sigma^{\mu} \partial_{\mu} \\
i \bar{\sigma}^{\mu} \partial_{\mu} & -\partial_{z}
\end{array}\right) \quad Q=\left(\begin{array}{cc}
0 & 2 q_{1} i \sigma^{2} \\
-2 q_{2} i \sigma^{2} & 0
\end{array}\right)
$$

whereupon (4.67) become

$$
D \Sigma=M a \Sigma+\delta\left(z+l_{1}\right) Q \Sigma^{*} .
$$

The second term on the RHS seems to complicate matters, but it is easy to reinterpret as a shift in the boundary condition on $\Sigma$.

To find this shift, we must first address the $z$-parity of the fermion on the orbifold branes. The bulk Dirac mass term must be odd on each orbifold $z_{o}$, and the fermion must satisfy

$$
i \gamma^{5} \Sigma\left(z_{o}-\epsilon\right)= \pm \Sigma\left(z_{o}+\epsilon\right)
$$

The signs can be chosen independently on each orbifold and different choices represent different superselection sectors. The four sectors can be classified by the (left, right) pairs $(++),(+-),(-+)$ and $(--)$. For definiteness in what follows we will consider $(++)$ and $(+-)$ cases. We will see that the former corresponds to twisted fermions in the bulk. The other two combinations are similar to these two with the interchange $Z \leftrightarrow X$. Hence if the parity assignment on an orbifold brane is $e^{i n \pi}$,

$$
Z\left(z_{o}-\epsilon\right)=e^{i n \pi} Z\left(z_{o}+\epsilon\right) \quad X\left(z_{o}-\epsilon\right)=-e^{i n \pi} X\left(z_{o}+\epsilon\right) .
$$

Then by Gaussian pillbox integration of (4.69) around the left IR (+) brane we obtain fermion boundary conditions there:

$$
q_{1} X\left(-l_{1}\right)=0 \quad X\left(-l_{1}\right)=q_{2} i \sigma^{2} Z^{*}\left(-l_{1}\right) .
$$

If both $q_{1}, q_{2} \neq 0$, then $Z\left(-l_{1}\right)=X\left(-l_{1}\right)=0$, which has no nonzero solutions. We will therefore choose $q_{1}=0$. Near the right IR brane there are no SUSY-breaking terms in the action, implying $X\left(l_{2}\right)=0$ for the $(++)$ case, and $Z\left(l_{2}\right)=0$ in the $(+-)$ case. On 
the UV brane we demand continuity of the wavefunction, since we do not impose orbifold boundary conditions there. The equations of motion and boundary conditions become:

$$
\begin{array}{ll}
D \Sigma=M a \Sigma & \\
X\left(-l_{1}\right)=q_{2} i \sigma^{2} Z^{*}\left(-l_{1}\right) & \\
Z\left(0_{+}\right)=Z\left(0_{-}\right) & X\left(0_{+}\right)=X\left(0_{-}\right) \\
X\left(l_{2}\right)=0 \quad(++) \text { case } & Z\left(l_{2}\right)=0 \quad(+-) \text { case }
\end{array}
$$

The appearance of the complex conjugate of the fermion field in the boundary condition of the left IR brane relates the positive and negative energy solutions there. We now turn to determining the spectrum of its solutions.

First, the system (4.73) does not admit any $4 D$ zero modes. Such modes would satisfy $\gamma^{\mu} \partial_{\mu} \Sigma=0$. The bulk equation would then reduce to the system

$$
Z^{\prime}=M a Z \quad X^{\prime}=-M a X
$$

Defining $w=m(|z|+L)$ (4.4), $\nu=M L$ (note the difference between this and (4.8)), the solutions are

$$
Z=\left\{\begin{array}{llll}
Z_{0}{ }_{L} w^{-\nu} & z<0 \\
Z_{0} & R & w^{\nu} & z>0
\end{array} \quad X= \begin{cases}X_{0} L & w^{\nu} \\
X_{0} R & z<0\end{cases}\right.
$$

where $Z_{0 L, R}, X_{0 L, R}$ are constant chiral spinors. Note the manifestation of the action of parity about the UV brane: under $z \leftrightarrow-z$, we must interchange $Z \leftrightarrow X$. This should be contrasted with the orbifold boundary conditions (4.71). This also clearly shows why $(++)$ case corresponds to twisted fermions and (+-) to normal ones. If we impose boundary conditions in the former case, we have different functions on different sides $\left(\sim w^{\nu}\right.$ to the left and $\sim w^{-\nu}$ to the right). In the latter case, the boundary conditions are imposed on the same function $\left(\sim w^{\nu}\right)$.

Inserting (4.75) into (4.73), we find that the only solutions are $Z_{0}{ }_{L}=Z_{0}{ }_{R}=X_{0} L=$ $X_{0 R}=0$. This differs from the single-throat situation in [39]. There, both the UV and IR branes were endowed with orbifold boundary conditions. Hence one of the chiral spinors always satisfied the boundary conditions trivially, allowing for an arbitrary constant profile in the bulk. This gave rise to a fermion zero mode in the effective $4 D$ theory.

Now we consider massive $4 D$ modes. The linear equation (4.73) can be diagonalized by squaring the operator $D$, as $D^{2}=\partial_{z}^{2}-\partial_{4}^{2}$ is diagonal in spinor indices. $\Sigma$ satisfies 
$\left(D^{2}-M^{2} a^{2}\right) \Sigma=M a^{\prime} i \gamma^{5} \Sigma$, which is diagonal in the spinor representation. Set $\Sigma=$ $\Sigma(z) \exp (-i p \cdot x)$, so that $\partial_{4}^{2} \Sigma=-m^{2} \Sigma$; and set $w=m(|z|+L)$ and $\nu=M L$. We find:

$$
\begin{array}{r}
\frac{d^{2} Z}{d w^{2}}+Z= \begin{cases}\frac{\nu(\nu+1)}{w^{2}} Z & z<0 \\
\frac{\nu(\nu-1)}{w^{2}} Z & z>0\end{cases} \\
\frac{d^{2} X}{d w^{2}}+X= \begin{cases}\frac{\nu(\nu-1)}{w^{2}} X & z<0 \\
\frac{\nu(\nu+1)}{w^{2}} X & z>0\end{cases}
\end{array}
$$

These are just Bessel equations. Before proceeding let us contrast this case to that when UV brane is an orbifold [39]. In the latter case $M$, and therefore $\nu=M L$, changes sign across the UV brane, ensuring that the potential on the two sides of the UV brane does not change. In our problem the Dirac mass is continuous across the UV brane; so the bulk potential for the chiral spinors changes across the brane, as is manifest in (4.76). Therefore the solutions on the right side are obtained from those on the left by $z \leftrightarrow-z, Z \leftrightarrow X$, so we need only explicitly solve for $z<0$. As a result the $(++)$ boundary conditions break the parity symmetry of (2.1), since they twist the fermion modes, while the $(+-)$ boundary conditions are parity-invariant.

We also note that the form of (4.76) shows that there have to be two sfermions with bulk masses related to the bulk fermion masses by $\sqrt{4+M_{B}^{2} L^{2}}=M_{F} L \pm 1 / 2$; only in this case would the bulk equations for both bosons and fermions admit the same spectrum. Furthermore, the boson boundary conditions must be chosen appropriately so that they reproduce the mass spectrum of the fermions. In what follows we will therefore assume that the spectrum of sfermions coincides with that of fermions in the SUSY limit, and that SUSY is broken exclusively by the $\sim q_{2}$ term.

Now, the solutions for $z<0$ are:

$$
\begin{aligned}
& Z=\sqrt{w}\left(A J_{\nu+1 / 2}(w)+B J_{-\nu-1 / 2}(w)\right) \\
& X=\sqrt{w}\left(C J_{\nu-1 / 2}(w)+D J_{-\nu+1 / 2}(w)\right) .
\end{aligned}
$$

The constant spinors $A, B, C, D$ are not all independent due to the equation of motion in (4.73) for $\Sigma$. In the rest frame of the massive modes, (4.73) becomes

$$
\left(\begin{array}{cc}
-\partial_{w} & 1 \\
1 & \partial_{w}
\end{array}\right) \Sigma=\frac{\nu}{w} \Sigma
$$


Upon substituting (4.77), we find $C=A, D=-B$. Using the map $z \leftrightarrow-z, Z \leftrightarrow X$, the bulk fermion wavefunctions in four-component notation are

$$
\begin{array}{ll}
\Sigma_{L}=\sqrt{w}\left(\begin{array}{c}
A_{L} J_{\nu+1 / 2}(w)+B_{L} J_{-\nu-1 / 2}(w) \\
A_{L} J_{\nu-1 / 2}(w)-B_{L} J_{-\nu+1 / 2}(w)
\end{array}\right) & z<0 \\
\Sigma_{R}=\sqrt{w}\left(\begin{array}{c}
A_{R} J_{\nu-1 / 2}(w)-B_{R} J_{-\nu+1 / 2}(w) \\
A_{R} J_{\nu+1 / 2}(w)+B_{R} J_{-\nu-1 / 2}(w)
\end{array}\right) & z>0
\end{array}
$$

where $A_{L, R}, B_{L, R}$ are constant, momentum-dependent 2-component spinors encoding $4 D$ fermion helicities (and are not $c$-numbers, in contrast to [39]). In an arbitrary frame, the basis spinors are given by Lorentz boosts of these.

To determine the spectrum we substitute the solution (4.79) into the boundary conditions specified in (4.73). We will use the shorthand notation $j_{\alpha, k}=J_{\alpha}\left(m\left(l_{k}+L\right)\right)$, $J_{\alpha}=J_{\alpha}(m L)$ and $J_{\alpha}\left(-m\left(l_{k}+L\right)\right)=\exp (2 \pi i \alpha) j_{\alpha, k}$. The analysis of the boundary conditions on the right IR brane and on the UV brane is straightforward; analysis of the boundary condition on the left IR brane is more subtle. As the latter involves $i \sigma^{2} Z^{*}\left(-l_{1}\right)$, in the momentum basis the mode which appears under complex conjugation is the negative energy mode. Defining the phase $\theta=2 \pi \nu$, we find:

$$
\begin{aligned}
& A_{L} j_{\nu-1 / 2,1}-B_{L} j_{-\nu+1 / 2,1}=-q_{2} i \sigma^{2} A_{L c}^{*} e^{-i \theta} j_{\nu+1 / 2,1}-q_{2} i \sigma^{2} B_{L}^{*} e^{i \theta} j_{-\nu-1 / 2,1} \\
& A_{L c} e^{i \theta} j_{\nu-1 / 2,1}+B_{L c} e^{-i \theta} j_{-\nu+1 / 2,1}=-q_{2} i \sigma^{2} A_{L}^{*} j_{\nu+1 / 2,1}-q_{2} i \sigma^{2} B_{L}^{*} j_{-\nu-1 / 2,1} .
\end{aligned}
$$

The remaining two boundary conditions then give

$$
\begin{gathered}
B_{R}=-\frac{j_{\nu+1 / 2,2}}{j_{-\nu-1 / 2,2}} A_{R} \quad(++) \text { case } \\
A_{L}\left(\begin{array}{c}
J_{\nu+1 / 2} \\
J_{\nu-1 / 2}
\end{array}\right)+B_{L}\left(\begin{array}{c}
J_{-\nu-1 / 2} \\
-J_{-\nu+1 / 2}
\end{array}\right)=A_{R}\left(\begin{array}{c}
j_{\nu-1 / 2,2} \\
J_{\nu+1 / 2}
\end{array}\right)+A_{R}\left(\begin{array}{c}
-J_{-\nu+1 / 2} \\
J_{-\nu-1 / 2}
\end{array}\right),
\end{gathered}
$$

and two more equations where $A_{L, R}^{c}, B_{L, R}^{c}$ replace $A_{L, R}, B_{L, R}$, and $m \rightarrow-m$. Here the superscript $c$ denotes the negative energy mode components, or charge conjugates. Note that if $q_{2}$ were zero, the $(+-)$ boundary conditions involve the same functions of $m l$ on both the left and right sides, while $(++)$ do not, again showing that the latter are twisted fermions. In either case there are eight boundary conditions for nine integration parameters: eight constant spinors $A_{L, R}, B_{L, R}, A_{L, R} c, B_{L, R} c$ and the $4 D$ mass $m$. One of the spinors is fixed by the overall normalization; for the remaining seven spinors the mass must be selected to solve the eight boundary conditions, wherefore the spectrum is discrete. 
We can solve this system of equations explicitly, expressing all the spinors in terms of $A_{L}$ which we choose by overall normalization for convenience. The masses are determined by the roots of the equation:

$$
\begin{aligned}
& \eta_{1}\left(j_{\nu-1 / 2,1} \mp q_{2} j_{\nu+1 / 2,1}\right)=\eta_{2}\left(j_{-\nu+1 / 2,1} \pm q_{2} j_{-\nu-1 / 2,1}\right) \\
& \eta_{1}=J_{-\nu+1 / 2} J_{\nu-1 / 2}+J_{\nu+1 / 2} J_{-\nu-1 / 2} \pm\left(J_{-\nu+1 / 2}^{2}-J_{-\nu-1 / 2}^{2}\right) \frac{j_{\nu \pm 1 / 2,2}}{j_{-\nu \mp 1 / 2,2}} \\
& \eta_{2}=J_{\nu-1 / 2}^{2}-J_{\nu+1 / 2}^{2} \pm\left(J_{-\nu+1 / 2} J_{\nu-1 / 2}+J_{\nu+1 / 2} J_{-\nu-1 / 2}\right) \frac{j_{\nu \pm 1 / 2,2}}{j_{-\nu \mp 1 / 2,2}}
\end{aligned}
$$

In the first of these equations the \pm sign changes for different chiralities, whereas in the latter two equations it changes with the boundary condition choices, being + for the $(++)$ case and - for the $(+-)$ case.

To extract the physical properties of the spectrum from this quagmire, we first replace $L$ by $\zeta L$ everywhere in these equations and then take a double limit $q_{2}=0, \zeta \rightarrow 0$, keeping $m l_{1}, m l_{2}$ fixed. This corresponds to removing SUSY breaking terms from the IR brane and (following the discussion at the beginning of Sec. 2) removing the UV brane, separating the two throats by an infinite distance. In this limit, the spectrum splits into separate towers of left- and right-localized modes. These modes modes will not be degenerate, since (2.1) is not symmetric. Using the small argument expansion of Bessel functions $J_{\alpha}(m \delta L) \sim(m \zeta L)^{\alpha}$, we see that to leading order $\eta_{1} \sim(m \zeta L)^{-2 \nu-1}$ is divergent, while $\eta_{2} \sim \mathcal{O}(1)$. Hence analyticity requires that the divergent part of the $\eta_{1}$ term must vanish, leading to the eigenvalue equations $J_{\nu-1 / 2}\left(m l_{1}\right) J_{\nu \pm 1 / 2}\left(m l_{2}\right)=0$, where we ignore terms $\mathcal{O}\left(L / l_{1}, L / l_{2}\right)<<1$. Therefore, the bulk fermion states split into two towers with masses given by the roots of

$$
\begin{array}{ll}
J_{\nu-1 / 2}\left(m l_{1}\right)=0 & \text { left }- \text { localized states } \\
J_{\nu \pm 1 / 2}\left(m l_{2}\right)=0 & \text { right }- \text { localized states }
\end{array}
$$

Let us check these conditions. Consider the solution (4.79). The $\sim B_{L, R}$ terms diverge in the limit $\zeta \rightarrow 0$; normalizability of the solution requires $B_{L, R}=0$ in that limit. The boundary condition on the UV brane is then satisfied. Moreover, when SUSY is unbroken, $q_{2}=0$ and the boundary conditions on either IR brane are $X=0$. On the left this gives precisely $J_{\nu-1 / 2}\left(m l_{1}\right)=0$ while on the right for the $(+ \pm)$ cases it gives $J_{\nu \pm 1 / 2}\left(m l_{2}\right)=0$.

When supersymmetry breaking is turned on and $\zeta=1$, the masses of these states get contributions from both supersymmetric UV effects and from SUSY-breaking effects. As 
we have explained above, we only wish to compute the SUSY-breaking induced mass shifts to leading order in the (dimensionless) SUSY breaking parameter $q_{2}$. For left-localized states, Eq. (4.82) implies that, to leading order, the SUSY-breaking induced splittings are determined by the roots of

$$
j_{\nu-1 / 2,1}= \pm q_{2} j_{\nu+1 / 2,1}
$$

Therefore, using the large-argument form of the Bessel functions $J_{\nu-1 / 2}(x) \sim \sqrt{\frac{2}{\pi x}} \cos (x-$ $\nu \pi / 2$ ), we find that (4.84) reduces to $\cos \left[m\left(l_{1}+L\right)-\nu \pi / 2\right]= \pm \mathcal{O}(1) q_{2}$. Thus the SUSY masses of light states are

$$
m \sim\left[\frac{(\nu-1) \pi}{2}+n \pi\right] \frac{1}{l_{1}},
$$

and the SUSY-breaking mass splittings are

$$
\delta m_{L}= \pm \mathcal{O}(1) \frac{q_{2}}{l_{1}}
$$

for both the $(++)$ and $(+-)$ cases. Therefore SUSY breaking lifts the degeneracy between chiral fermions.

To compute the transmission of SUSY breaking to the right wall, we need to determine the couplings. Naively, the fermion boundary conditions (4.73) might suggest that the $(++)$ case does not give an efficient SUSY breaking mechanism, because $X=0$ on the right wall and there is no manifest Majorana mass for $Z$ in that case. However the $z$-derivative terms in (4.66) give Majorana masses upon Kaluza-Klein reduction, with the same order of magnitude as the manifest $Z$ Majorana masses. Then from (4.80), (4.81) we can deduce that for these states $A_{R}, B_{R}, B_{L} \sim(m L)^{2 \nu} A_{L}$ and so these modes are dominated by the $\sim A_{L}$ component in (4.79) throughout most of the bulk geometry. Because $l_{1}>l_{2}$, the proper normalization of the wavefunction is achieved by setting $A_{L} \sim 1 / \sqrt{l_{1}}$ paralleling the case of bosons from $\S 4$. Therefore $A_{R} \sim(m L)^{2 \nu} / \sqrt{l_{1}}$. Recalling the form of the fermionic interaction Lagrangian (4.42) and the conformal scalings of the fields $\phi_{4 D}=a \phi_{b}$, $\lambda_{4 D b}=a^{3 / 2} \lambda_{b}$ and $\lambda_{4 D B}=a^{2} \lambda_{B}=a^{2} \Sigma$, the effective $4 D$ couplings are the same as that for the bosons. If $g^{I}{ }_{J}=\mathcal{O}(1) / \sqrt{M_{5}}$, the left-localized fermion couplings to the fields on the right IR brane are

$$
\begin{aligned}
g^{L}{ }_{4 D} & =\frac{\mathcal{O}(1)}{\sqrt{M_{5} L}} \\
g_{R 4 D}^{L} & =\frac{\mathcal{O}(1)}{\sqrt{M_{5} L}} \sqrt{\frac{l_{2}}{l_{1}}}(m L)^{2 \nu} .
\end{aligned}
$$


For heavy fermions, the tunneling suppression weakens, and eventually disappears for the states with $m L>1$. The masses and the couplings of these states behave essentially as those for bosons, in eqs. (4.25), (4.26).

Now we look at the right-localized states and compute their mass splitting and $4 D$ effective theory couplings. For these states, $A_{L}, B_{L}, B_{R} \sim(m L)^{2 \nu} A_{R}$ and so the correct normalization requires $A_{R} \sim 1 / \sqrt{l_{2}+(m L)^{4 \nu} l_{1}}$. The eigenvalue equation becomes

$$
j_{\nu \pm 1 / 2,2}=\mathcal{O}(1)(m L)^{2 \nu+1} \mp q_{2} \mathcal{O}(1)(m L)^{4 \nu}
$$

and to leading order the supersymmeric masses and the SUSY-breaking induced mass splittings are

$$
\begin{gathered}
m \sim\left[\frac{(\nu-1) \pi}{2}+n \pi\right] \frac{1}{l_{2}} \\
\delta m= \pm \mathcal{O}(1) \frac{q_{2}}{l_{2}}(m L)^{4 \nu} .
\end{gathered}
$$

The effective $4 D$ couplings of the right-localized fermions are

$$
g_{i 4 D}^{R} \sim g_{i}^{R}\left(A_{i} / A_{R}\right) / \sqrt{a l} \sim g_{i}^{R}\left(A_{i} / A_{R}\right) / \sqrt{L},
$$

so that

$$
\begin{aligned}
g_{L 4 D}^{R} & =\frac{\mathcal{O}(1)}{\sqrt{M_{5} L}}(m L)^{2 \nu} \sqrt{\frac{l_{1}}{l_{2}+(m L)^{4 \nu} l_{1}}} \\
g_{R 4 D}^{R} & =\frac{\mathcal{O}(1)}{\sqrt{M_{5} L}} \sqrt{\frac{l_{2}}{l_{2}+(m L)^{4 \nu} l_{1}}} .
\end{aligned}
$$

Now we are ready to compute the SUSY breaking transmission to the fields localized on the right IR brane.

As a warmup, we consider the radiatively-induced scalar masses. We begin with terms $\sim m_{s}^{2} \phi_{b}^{\dagger} \phi_{b}$, for any brane scalars $\phi_{b}$ that may have direct couplings to the split bulk multiplets. To compute the masses, we again use the formulas (4.48) and (4.54) derived in $§ 4.4$. Using (4.86), (4.87), (4.90) and (4.91), we see that for the left-localized modes, $F=x^{4 \nu} l_{2} / l_{1}, \beta_{B}-\beta_{F}=q_{2}$ and $l=l_{1}$. Therefore the scalar masses induced by the left-localized modes are

$$
m_{s}^{2} \sim \mathcal{O}(1) \frac{q_{2}}{M_{5} L} \frac{l_{2}}{l_{1}^{3}}\left(\frac{L}{l_{1}}\right)^{4 \nu}
$$

For the right-localized modes, $F=l_{2} /\left(l_{2}+x^{4 \nu} l_{1}\right), \beta_{B}-\beta_{F}=q_{2} x^{4 \nu}$ and $l=l_{2}$, and so

$$
m_{s}^{2} \sim \mathcal{O}(1) \frac{q_{2}}{M_{5} L} \frac{1}{l_{2}\left(l_{2}+\left(L / l_{2}\right)^{4 \nu} l_{1}\right)}\left(\frac{L}{l_{2}}\right)^{4 \nu} .
$$


Here again if $l_{1}<l_{2}\left(\frac{l_{2}}{L}\right)^{4 \nu}$, the limit is

$$
m_{s}^{2} \sim \mathcal{O}(1) \frac{q_{2}}{M_{5} L} \frac{1}{l_{2}^{2}}\left(\frac{L}{l_{2}}\right)^{4 \nu}
$$

When $l_{1}>l_{2}\left(\frac{l_{2}}{L}\right)^{4 \nu}$ the scalar masses are

$$
m_{s}^{2} \sim \mathcal{O}(1) \frac{q_{2}}{M_{5} L} \frac{1}{l_{2} l_{1}}
$$

Hence again the right-localized states give a dominant contribution to the scalar masses as long as $l_{1}>l_{2}$. Similar SUSY-breaking mass splittings are also induced for any other right IR-brane chiral supermultiplet, in particular for an adjoint multiplet. Because these terms contain bulk fermions with Majorana mass in the loop, they break $R$-symmetry.

We further consider SUSY breaking-induced masses of the form $m_{A}^{2} \phi_{b}^{2}$, which violate particle number conservation. Such terms arise for example in theories which contain boundary interactions

$$
\mathcal{L}_{\text {int }}=g_{5}^{2} \Phi_{b}^{2}(x)\left[\Phi_{B}^{\dagger}\left(x, z=l_{2}\right)\right]^{2}+\mu \Phi_{B}^{2}\left(x, z=-l_{1}\right),
$$

where the first term denotes the interaction between the right brane scalars and the bulk scalars while the second term stands for the SUSY-breaking left brane mass term for the bulk scalars. Note that the quartic interaction term can arise from the effective $4 D$ superpotential

$$
W=W_{S S M}+g \mathcal{F}_{b}^{3}+g \mathcal{F}_{b} \sum_{m} \mathcal{F}_{B}^{2}
$$

where $\phi_{b}$ and $\phi_{B}$ are the superfields containing $\Phi_{b}$ and $\Phi_{B}$. To proceed, we need to reduce (4.96) to $4 \mathrm{~d}$, and determine the terms it gives rise to in the effective $4 \mathrm{~d}$ theory. With the usual rules for dimensional rescaling for the KK modes, and the normalizations of bulk wavefunctions which specify the overlap, we find that the effective $4 \mathrm{~d}$ theory is

$$
\mathcal{L}_{\text {eff }}=g_{4}^{2} \phi_{b}^{2}\left[\phi^{\dagger}\right]_{B}^{2}+\delta m_{B}^{2} \phi_{B}^{2}
$$

where the couplings and mass insertions for the left localized modes are $g_{4 D}^{2} \sim$ $\frac{l_{2}}{M_{5} L l_{1}}(m L)^{4 \nu}$ and $\delta m_{B}^{2} \sim 1 / l_{1}^{2}$ for light modes and vanishingly small $\delta M_{B}^{2}$ for heavy modes. For right-localized modes, $g_{4 D}^{2} \sim \frac{1}{M_{5} L}$ while $\delta m_{B}^{2} \sim \frac{q_{2}}{l_{1}\left(l_{2}+(m L)^{4 \nu} l_{1}\right)}(m L)^{4 \nu}$ for light modes and again vanishingly small $\delta m_{B}^{2}$ for heavy modes. These vertices give rise to a 
one-loop Feynman diagram with $\phi_{b}$ as external legs and $\phi_{B}$ loop, and one mass insertion $\sim m_{B}^{2}$, which generates the one-loop mass term $\sim m_{A}^{2} \phi_{b}^{2}$ where

$$
m_{A}^{2} \sim \mathcal{O}(1) \sum_{m} g^{2} \delta m_{B}^{2} \int \frac{d^{4} p}{\left(p^{2}+m_{B}\right)^{2}}
$$

It is now clear that the right-localized modes dominate the contribution to (4.99). We can now evaluate the contribution of the left-localized modes by our summation technique, noticing that by defining $F=g^{2} \delta m_{B}^{2} M_{5} L$ and $\left(p^{2}+m_{B}\right)^{-2}=-\left.p^{-2} \partial_{s}\left(s p^{2}+m_{B}\right)^{-1}\right|_{s=1}$ we get

$$
m_{A}^{2} \sim-\left.\frac{\mathcal{O}(1)}{M_{5} L} \partial_{s} \int \frac{d^{4} p}{p^{2}} \sum_{m} \frac{F}{s p^{2}+m_{B}}\right|_{s=1} .
$$

After summing over the KK masses and performing the angular integrals, we obtain to leading order

$$
m_{A}^{2} \sim \frac{\mathcal{O}(1) l_{2}}{M_{5} L} \int d p(F(i \sqrt{s} p L)+F(-i \sqrt{s} p L)),
$$

where we have used eqs. (4.51) and (4.52), and ignored the exponentially suppressed contributions. Using (4.34), (4.39), (4.40), (4.41) and (4.44), we can approximate $F$ by

$$
F=\mathcal{O}(1) \frac{q_{2}}{l_{1}\left(l_{2}+(m L)^{4 \nu} l_{1}\right)}(m L)^{4 \nu}\left(1-\theta\left(|m|-\frac{q_{2}}{l_{2}}\right)\right),
$$

and upon substituting into (4.101) we find

$$
m_{A}^{2} \sim \mathcal{O}(1) \frac{q_{2}}{M_{5} L} \frac{1}{l_{1}\left(l_{2}+\left(L / l_{2}\right)^{4 \nu} l_{1}\right)}\left(\frac{L}{l_{2}}\right)^{4 \nu} .
$$

Hence this mass is parametrically similar to (4.93).

Now we turn to the gaugino masses induced by the transmission of SUSY breaking. To lowest order, the gaugino masses must come from the loop diagram involving a bulk multiplet with an $R$-symmetry breaking mass term. Because the gauge fields live only on the brane and not in the bulk, gauge invariance requires the existence of an adjoint which couples to the gaugino through terms like $\sim g \operatorname{Tr}\left(\Phi_{b} \lambda_{b}\right) \lambda_{B}$. In order not to spoil gauge unification, the adjoint must be heavy, $M \sim 1 / L$. The adjoint must also acquire number operator-breaking mass term, in order to give rise to the gaugino mass. This is clear from diagram topology, since the "in"-vertex is generated by the term $\sim \operatorname{Tr}\left(\Phi_{b} \lambda_{b}\right) \lambda_{B}$, and the Majorana mass insertion $m_{M} \lambda_{B}^{\dagger} \lambda_{B}^{\dagger}$ on the $\lambda_{B}$ line converts it into $\lambda_{B}^{\dagger}$, and so the "out"-vertex must be $\sim \operatorname{Tr}\left(\Phi_{b} \lambda_{b}\right) \lambda_{B}$ to contract this with. Hence the adjoint must pick 
up a mass term $\sim \delta m_{A}^{2} \Phi_{b} \Phi_{b}$ so that the contraction does not vanish. Such a term arises radiatively, as given in eq. (4.103).

Next we must reduce the Majorana mass term to the $4 \mathrm{~d}$ effective theory. The left- and right-localized fermions acquire hierarchically different Majorana masses in four dimensions but they also have very different couplings to the fields on the right wall. Indeed, for fermions $\lambda_{B} 4 D=a^{2} \lambda_{B}$, so the Majorana mass for left-localized fermions is $a^{4} q_{2}\left[\chi^{T} i \sigma^{2} \chi+\right.$ h.c. $]=q_{2}\left[X^{T} i \sigma^{2} X+h . c.\right] \sim\left(q_{2} / l_{1}\right)\left[\psi_{4 D}^{T} i \sigma^{2} \psi_{4 D}+h . c.\right]$, i.e. it is $q_{2} / l_{1}$ in the $4 D$ theory. For the right-localized fermions, there is an additional suppression since $A_{L} \sim(m L)^{2 \nu} A_{R}$ is the parameter which sets the scale of the wavefunction on the left wall. Therefore the rightlocalized fermion has tunneling-suppressed $4 D$ Majorana mass, $q_{2} f^{2}(m L) /\left(l_{2}+f^{2}(m L) l_{1}\right)$. It is small for the light states, but approaches that of the left-localized bulk fermions for the heavy modes.

Define

$$
\tilde{q}=\left(q_{2} / l_{1}, q_{2} f^{2}(m L) /\left(l_{2}+f^{2}(m L) l_{1}\right)\right)
$$

for the left- and right-localized fermions, respectively. The gaugino mass transmitted to the right IR brane is, approximately,

$$
m_{g} \sim \mathcal{O}(1) \sum_{m} \tilde{q} \delta m_{A}^{2} g^{2} \int \frac{d^{4} p}{\left(p^{2}+M^{2}\right)^{2}\left(p^{2}+m^{2}\right)} .
$$

Here $\delta m_{A}^{2}$ is the radiative mass correction of the adjoint, given in eq. (4.93), and $M \sim L^{-1}$ is the mass of the adjoint. We sum the series using (4.51). Here $F=M_{5} L \tilde{q} g^{2} \delta m_{A}^{2}$. After doing the angular integrals, the gaugino mass is approximately

$$
\begin{aligned}
m_{g} & \sim \frac{\mathcal{O}(1) l}{\alpha M_{5} L} \int_{0}^{\infty} \frac{d p p^{2}}{\left(p^{2}+M^{2}\right)^{2}}\left(\frac{1}{2}[F(i p L)+F(-i p L)]\right. \\
& \left.+\frac{F(i p L)}{e^{2 \pi l p / \alpha-2 i \pi \beta(i p L)}-1}+\frac{F(-i p L)}{e^{2 \pi l p / \alpha+2 i \pi \beta(-i p L)}-1}\right) .
\end{aligned}
$$

The integral is finite, because it is cut off in the UV where $F \rightarrow$ const. and the integrand is $\sim 1 / p^{2}$. We can ignore the terms with exponential suppressions in the UV because the main contribution to them comes from the momenta $p \sim \alpha / 2 \pi l$, while the main contribution to the unsuppressed integrals comes from $p \sim M$, such that their ratio is $\sim 1 /(M l)^{3}<<1$. Thus using the saddle point method to estimate the integral, we obtain

$$
m_{g} \sim \frac{\mathcal{O}(1) l}{\alpha M M_{5} L}(F(i M L)+F(-i M L))
$$


Now, for either the left- or the right-localized modes, the states with high loop momentum $\sim M$ dominate (4.106). Since $f^{2}(M L) \sim 1$, the relevant bulk fermion Majorana mass is always $\tilde{q}=q_{2} /\left(l_{1}+l_{2}\right) \sim \mathcal{O}(1) q_{2} / l_{1}$. Also, in this limit the couplings approach rapidly those of eq. (4.26). In particular, the left-localized modes couple to the right IR brane with $g^{2} \sim\left(M_{5} L\right)^{-1} l_{2} / l_{1}$, while the right-localized modes couple more strongly, with $g^{2} \sim$ $\left(M_{5} L\right)^{-1}$. This shows clearly that this integral does not give any tunneling suppression factors to the gaugino mass, which comes entirely from the tunneling suppression factors in $\delta m_{A}^{2}$. So tunneling suppression terms are one-loop effects.

Therefore, for the left-localized modes, $F=q_{2} \delta m_{A}^{2} l_{2} / l_{1}^{2}$ and $l=l_{1}$ (4.85), (4.87), so using (4.103) for $\delta m_{A}^{2}$,

$$
m_{g} \sim \mathcal{O}(1)\left(\frac{q_{2}}{M_{5} L}\right)^{2} \frac{1}{M l_{1}} \frac{1}{l_{2}+\left(L / l_{2}\right)^{4 \nu} l_{1}}\left(\frac{L}{l_{2}}\right)^{4 \nu}
$$

For the right-localized modes, $F=q_{2} \delta m_{A}^{2} / l_{1}$ and $l=l_{2}$ (4.89),(4.87), and so their contribution to the gaugino mass is essentially the same as (4.107). Both contributions are dominated by the states with loop momenta of the order of the adjoint mass, $p \sim M$. As before, we have two regimes, depending on $\left(L / l_{2}\right)^{4 \nu} l_{1} / l_{2}$.

The formula for the gaugino mass generated by loops of the bulk fields is:

$$
m_{g} \sim \mathcal{O}(1)\left(\frac{q_{2}}{M_{5} L}\right)^{2} \frac{1}{M l_{1}} \times \begin{cases}\frac{1}{l_{2}}\left(\frac{L}{l_{2}}\right)^{4 \nu} & l_{1}<l_{2}\left(\frac{l_{2}}{L}\right)^{4 \nu} \\ \frac{1}{l_{1}} & l_{1}>l_{2}\left(\frac{l_{2}}{L}\right)^{4 \nu}\end{cases}
$$

The tunneling suppression is manifest in the form of $(L / l)^{4 \nu}$ dependence, and begins to disappear in the limit where the localization of bulk states is weak.

For comparison with other mediation mechanisms, we also consider the corrections to the gaugino mass from gravity and Weyl anomaly mediation. The gravity mediation would give gaugino mass of order of teh gravition mass $m_{3 / 2}$ [25]. The anomaly-mediated supersymmetry breaking leads to a universal form of the gaugino mass $30, m_{q}^{A}=\frac{\beta_{g}\left(g^{2}\right)}{g^{2}} m_{3 / 2}$, where $g$ is the gauge coupling, $\beta_{g}$ the gauge coupling $\beta$-function. The gravitino mass is generated by the exchange of split bulk multiplets in the loop. By tree-level Ward identities, the mass formula is, approximately,

$$
m_{3 / 2} \sim \mathcal{O}(1) \sum_{m} \frac{\tilde{q} \delta m_{B}^{2}}{M_{4}^{2}} \int d^{4} p \frac{p^{2}}{\left(p^{2}+m^{2}\right)^{3}},
$$


where $\delta m_{B}^{2}$ is the bulk boson $R$-symmetry breaking mass. We can again sum over the bulk mode masses using the technique in $\S 4$. Since the $R$-symmetry breaking terms $\tilde{q} \delta m_{B}^{2}$ depend on the tunneling suppression, the left-localized mode contributions will dominate. The left-localized fermion Majorana mass is $\tilde{q} \sim q_{2} / l_{1}$, while the bulk boson $R$-breaking mass squared is $\delta m_{B}^{2}=F / l_{1}^{2}$, where $F$ is $\mathcal{O}(1)$ for light states and and decreases with mass as $F \sim q_{2} /\left(l_{1} m\right)$ for heavy states. For simplicity we again approximate it by the step function $F=1-\theta\left(|m|-q_{2} / l_{1}\right)$. Using this, noting that $p^{4} /\left(p^{2}+m^{2}\right)^{3}=(1 / 2) \partial_{s}^{2}\left(s p^{2}+\right.$ $\left.m^{2}\right)^{-1}$ and recalling (4.53), where we can now ignore the subleading terms with exponential momentum suppressions, we find

$$
m_{3 / 2} \sim \mathcal{O}(1) \frac{q_{2}}{M_{4}^{2} l_{1}^{2}} \partial_{s}^{2} s^{-1 / 2} \int_{0}^{\infty} d p\left(1-\theta\left(|p|-q_{2} / l_{1}\right)\right)
$$

Therefore the gravitino mass is, using $l=l_{1}$ for the left-localized modes,

$$
m_{3 / 2} \sim \mathcal{O}(1) \frac{q_{2}}{M_{4}^{2} l_{1}^{3}}
$$

This mode is lighter than other gravitino KK modes, whose masses are $\sim l_{1}^{-1}, l_{2}^{-1}$, because it is protected by SUSY. So tunneling mediation will be a dominant mechanism for transmitting SUSY-breaking mass splittings to the visible sector as long as $m_{g}>m_{3 / 2}$. We address the conditions when this is satisfied below.

\section{Phenomenology of Tunneling Mediation}

The SSM cutoff is set by the conformal distance of the SSM to the UV brane, $l_{2}^{-1} \sim 10^{15} \mathrm{GeV}$. This choice ensures that there is no fundamental obstacle to implement the supersymmetric unification of gauge couplings. Squark masses are generated by radiative corrections including gaugino loops. They start out close to zero in the UV, and rise via the RG flow in the IR. As a result, $m_{s q} \sim T e V$ at the electroweak energy and is comparable to the gaugino mass, as in no-scale models [40].

Tunnelling suppression produces large mass hierarchies without much effort. For example, take $M_{5} \sim 10^{16} \mathrm{GeV}, L \sim 5 / M_{5}, l_{2} \sim 5 L, M \sim 1 / L$ and $q_{2} \leq \mathcal{O}(1)$. The tunnelling suppression coefficient $\nu$ and the SUSY breaking scale $l_{1}^{-1}$ must be chosen so that $m_{g} \sim T e V$. For $\nu=1$, i.e. with little tunnelling suppression, the required SUSY breaking scale is low, $l_{1}^{-1} \sim 10^{10} \mathrm{GeV}$. This scale implies a micron-range gravitino mass 
$m_{3 / 2} \sim e V$. If $\nu=3$, the SUSY breaking scale should be $l_{1}^{-1} \sim 3 \times 10^{13} \mathrm{GeV}$, closer to the unification scale. The induced gravitino mass is $m_{3 / 2} \sim 270 \mathrm{GeV}$.

Does tunneling mediation solve the flavor problem? Just as in gravity mediation, the answer is, in general, negative. The reason is that although the squark masses vanish at precisely the GUT scale, they are non-zero an order of magnitude below $M_{G U T}$; the squark masses are "hard" - they do not vanish sufficiently rapidly in the UV. So they can be distorted by the nearby flavor physics, taking place at $M_{G U T}$ or $M_{P l}$, which is also responsible for the large intergenerational differences of quark and lepton masses. These distortions can cause a misalignment of squark and quark masses leading to unacceptable flavor violation. The only way to avoid this is for the UV theory to be special, e.g. the squark masses could line up with the quark masses and therefore not create new flavor violating angles.

There are also model-independent gravity [28,25] and anomaly [30] mediated contributions to the sparticle masses that are bounded by $m_{3 / 2}$. They are subdominant to the tunneling mediated contributions as long as

$$
l_{1}>l_{2}\left(\frac{l_{2}}{L}\right)^{2 \nu+1 / 2} \frac{1}{M_{4} l_{2}} .
$$

It is not difficult to satisfy this constraint because of the $4 \mathrm{~d}$ Planck mass $M_{4}$ in the denominator on the RHS.

The hierarchies we produce do not originate from the AdS scaling as in [8]. In our case the cutoff on the SSM brane is $M_{G U T}$. Furthermore, our effect would persist with slight modifications given any warp factor which raises a barrier between different throats.

\section{Tunneling, 4D Effective Field Theory, and Compactified AdS/CFT}

In this section we will discuss the four-dimensional description of our results, in the case that the throats are the near-horizon geometries of D3-branes. In this background, normalizable closed string excitations have a dual description as the massless gauge theory excitations on the D3-brane worldvolume [13]. More precisely, if one does not scale $\alpha^{\prime}$ strictly to zero, there are scattering states in the asymptotically flat region which are purely ingoing at the AdS horizon; and modes which are localized in the AdS throat, outgoing at the past horizon, and ingoing at the future horizon [41.42]. In the strict near horizon limit with $\alpha^{\prime} \rightarrow 0$, the latter become normalizable modes in AdS, which are dual to states of the D-brane CFT created by modes of local gauge-invariant operators $\mathcal{O}_{I}(x)$ 
[18,43,44. The former become non-normalizable modes which are dual to couplings $\lambda_{I}$ multiplying these operators [17, 18] in the dual CFT description:

$$
\int d^{4} x \lambda_{I} \mathcal{O}_{I}(x)
$$

We will refer to these modes as "asymptotic closed strings" in reference to the localization of their wavefunctions in the asymptotically flat region of the full D3-brane geometry.

We will focus on the five-dimensional graviton $h_{\mu \nu}$. When $h$ is polarized along the boundary, the normalizable and non-normalizable modes of $h$ are, respectively, dual to the modes of and couplings multiplying the gauge theory stress tensor $T_{\mu \nu}$.

When the AdS throat opens into some compact manifold, the low-energy states supported in this manifold become modes of four-dimensional (super)gravity plus additional fields arising from the compactification. These modes can be modeled as excitations on the UV ("Planck") brane, which couple to modes supported in the bulk of AdS [11]. We might expect to maintain the interpretation of these latter modes as excitations of a dual CFT with a cutoff, now coupled to four-dimensional (super)gravity [45]. In the setup of $\S 2$, the low-energy action would then include arbitrary products of gauge theory operators

$$
\delta S=\int d^{4} x \lambda_{i j} \mathcal{O}_{i}^{(1)} \mathcal{O}_{j}^{(2)}+\ldots
$$

where the superscripts $(1,2)$ label the left and right AdS regions of Fig. 2.

The tunneling calculation in $\S 2$ tests this proposal. An operator $\mathcal{O}$ of dimension $\Delta$ will appear in the action with powers of the UV cutoff $\Lambda_{U V}$ :

$$
\int d^{4} x \Lambda_{U V}^{4-\Delta} \lambda \mathcal{O}_{\Delta}
$$

where $\lambda$ is dimensionless. If $\mathcal{O}_{\Delta}$ takes the form (6.1), it will mediate transitions between modes of these operators with a probability:

$$
P \sim \lambda\left(\frac{m}{\Lambda_{U V}}\right)^{2 \Delta-8}
$$

and a transition rate:

$$
\Gamma \sim m \lambda\left(\frac{m}{\Lambda_{U V}}\right)^{2 \Delta-8}
$$

where $m$ is the mass of the initial state. The results of $\S 2$ for the tunneling of graviton modes give:

$$
P \sim m^{4} L^{4} \sim e^{-4 R_{1} / L}
$$


and

$$
\Gamma \sim \frac{1}{L} e^{-5 R_{1} / L}
$$

A glueball near the left IR brane has mass:

$$
m \sim \frac{1}{L} e^{-R_{1} / L}
$$

We conclude that the transition between glueballs is mediated by a dimension- 6 operator and that the natural UV cutoff is $\Lambda_{U V}=1 / L=M_{U V}$. . We note first that this is not the four- (or five- or ten-) dimensional Planck scale as would appear in the minimal coupling of gravity to a CFT. Instead, the dimensionful parameters are set by scales in the background geometry.

A further surprise is that the operator mediating the transition in $\S 2$ is not constructed from the CFTs alone. The $2 \times(\mathrm{CFT})$ operator mediating a transition between the gravitons $h_{\mu \nu}$ in the two AdS regions would be the dimension eight operator:

$$
: T_{\mu \nu}^{(1)} T_{\mu \nu}^{(2)}:
$$

However, the outgoing wave in the second throat has a wavefunction proportional to

$$
\psi \sim J_{2}+i N_{2}
$$

In the AdS/CFT dictionary this is a Fourier mode of the "bulk-boundary propagator" 17,41 and corresponds to a change in the coupling, i.e. an asymptotic closed string mode,

$$
\delta h_{\mu \nu} T_{\mu \nu}
$$

In field theory language, the calculation of $\S 2.1$ is computing the overlap

$$
\left\langle\psi_{1}\left|T_{\mu \nu}^{(1)} H^{(2) \mu \nu}\right| \psi_{2}\right\rangle
$$

4 There is some freedom in this identification of the cutoff. In the denominator of (2.1) we can replace $L$ with $\zeta L$. The tunnelling formula is the same, but with $\Lambda_{U V}=1 / \zeta L$. $\zeta$ will be fixed by the details of the microscopic model.

5 In a perturbative string limit, $M_{s}<M_{(p l) 4,5,10}$. If the throats were close enough, the strings stretched between the D-branes could play an appreciable role in the dynamics below the Planck scale. In the case at hand these "W bosons" are much heavier than $M_{s}$. 
$\left|\psi_{1}\right\rangle$ is the state on the left side of the wall, and $\left|\psi_{2}\right\rangle$ is the state with outgoing wavefunction $J+i N$ on the right. If this overlap is to be non-vanishing, $H^{(2)}$ must be built from asymptotic closed-string degrees of freedom.

There are many candidates for $H$. Obvious terms constructed from bulk gravitons are: $R_{\mu \nu}^{(5)}(z=0, x)$ and $g_{\mu \nu}^{(5)} R^{(5)}(z=0, x)$, where these fields are now included in the $4 \mathrm{~d}$ effective action. In addition, the "UV wall" defines a covariant normal vector $n_{\mu}$, whose covariant derivatives can be combined into an infinite number of dimension-two covariant two-index tensors. Two sets of terms are $(n \cdot \nabla)^{\alpha} K_{\mu \nu}(x)$ and $\nabla_{\mu} \nabla_{\nu}(n \cdot \nabla)^{\alpha} K$, where $K_{\mu \nu}$ is the extrinsic curvature of the UV wall.

Another sign that we must couple the CFT and asymptotic closed string operators is that in the standard RS picture, the mass eigenstates for $h$ take the form:

$$
\psi \sim J_{2}+b(m L)^{2} N_{2}
$$

where $b$ is of order 1 . When $L \rightarrow 0, J_{2}$ and $N_{2}$ become normalizable and non-normalizable, respectively. This implies that the stress tensor is modified:

$$
\tilde{T}_{\mu \nu}=T_{\mu \nu}^{\mathrm{CFT}}+b\left(L^{2} \partial^{2}\right) \frac{1}{L^{2}} I_{\mu \nu}
$$

where $I$ is a linear combination of dimension-two tensors constructed from the 10d graviton polarized along the D3-brane. The additional derivatives reflect the coefficient of $N$ in (6.10). Such a mixing will generically occur for all other operator modes as well. So the Hamiltonian mixes the CFT and asymptotic closed-string degrees of freedom; the effective theory includes operators from both sectors.

The small, derivative-suppressed coefficient in (6.10), (6.11) also suppresses the dimension-four operator one might expect from the purely asymptotic closed-string contribution to : $\tilde{T}^{(1)} \tilde{T}^{(2)}$ :. This derivative suppression also means that at sufficiently low energies the modes localized in one AdS throat are well described by the original CFT stress tensor. However, at best this decoupling works when studying a single throat. When coupling the two, we must include modes from both throats, and asymptotic closed string modes, even at low energy. We must take care with the scale-radius correspondence when the AdS throat is coupled to additional degrees of freedom. Low energy modes are supported in the compactification manifold or down the right throat as well as far down the left throat. Furthermore, if one chooses boundary conditions such that wavefunctions 
have larger mixings between $J$ and $N$, even the dynamics far down one AdS throat will cease to be well described by CFT dynamics.

The perturbations forced on us are distinct from another effect we might expect. A conformal field theory coupled to gravity will generically have all relevant (and irrelevant) CFT operators appearing in the Lagrangian, with coefficients given by suitable powers of the UV scale $M_{4}$. This is not the effect we are discussing here. As in all discussions of Randall-Sundrum scenarios, we have assumed the absence of any such relevant operators (tachyons satisfying the Breitenlohner-Freedman bound 46,17]); these would yield an instability of the gravity solution after introducing the UV brane.

\section{Acknowledgements}

It is a pleasure to thank N. Arkani-Hamed, S. Giddings, J. Maldacena, A. Peet, M. Peskin, J. Polchinski, A. Pomarol, M. Schmaltz, S. Shenker, P. Steinhardt, A. Strominger, L. Susskind, S. Thomas, H. Tye and H. Verlinde for interesting discussions about related subjects. This work was supported in part by the DOE under contract DE-AC03-76SF00515. During the completion of this project, the authors enjoyed the hospitality of the Institute for Theoretical Physics at Santa Barbara, and were supported by the National Science Foundation under grant number PHY-99-07949. A.L. would like to thank the Center for Geometry and Theoretical Physics at Duke University for its hospitality while this work was completed; he received additional support there from NSF grant DMS-0074072. The work of S.K. was supported in part by a Packard Fellowship for Science and Engineering and an Alfred P. Sloan Foundation Fellowship, and the work of E.S. was supported in part by a DOE OJI grant and an Alfred P. Sloan Foundation Fellowship. 


\section{References}

[1] H. Georgi and S.L. Glashow, Phys. Rev. Lett. 32 (1974) 438.

[2] S. Dimopoulos and H. Georgi, Nucl. Phys. B192 (1981) 150.

[3] P. Hořava and E. Witten, "Heterotic and Type I String Dynamics from ElevenDimensions," Nucl. Phys. B460 (1996) 506, hep-th/9510209.

[4] E. Witten, "Strong Coupling Expansion of Calabi-Yau Compactification," Nucl. Phys. B471 (1996) 135, hep-th/9602070.

[5] J. Dai, R. G. Leigh and J. Polchinski, Mod. Phys. Lett. A4, 2073 (1989); P. Horava, Phys. Lett. B231, 251 (1989); P. Horava, Nucl. Phys. B327, 461 (1989); G. Pradisi and A. Sagnotti, Phys. Lett. B216, 59 (1989); J. Polchinski, Phys. Rev. Lett. 75, 4724 (1995), hep-th/9510017.

[6] N. Arkani-Hamed, S. Dimopoulos and G. Dvali, Phys. Lett. B429 (1998) 263, hepth/9803315; I. Antoniadis, N. Arkani-Hamed, S. Dimopoulos and G. Dvali, Phys. Lett. B436 (1998) 257, hep-th/9804398; N. Arkani-Hamed, S. Dimopoulos and G. Dvali, Phys. Rev. D59 (1999) 086004, hep-th/9807344.

[7] N. Arkani-Hamed and S. Dimopoulos, hep-ph/9811353; N. Arkani-Hamed, S. Dimopoulos, G. Dvali and J. March-Russell, hep-ph/9811448; N. Arkani-Hamed and M. Schmaltz, Phys. Rev. D61 (2000) 033005, hep-ph/9903417; N. Arkani-Hamed, Y. Grossman and M. Schmaltz, Phys. Rev. D61 (2000) 115004, hep-ph/9909411.

[8] L. Randall and R. Sundrum, "A Large Mass Hierarchy from a Small Extra Dimension," Phys. Rev. Lett. 83 (1999) 3370, hep-th/9905221.

[9] E. Verlinde and H. Verlinde, JHEP 0005 (2000) 034; N. Arkani-Hamed, S. Dimopoulos, N. Kaloper and R. Sundrum, Phys. Lett. B480 (2000) 193, hep-th/0001197; S. Kachru, M. Schulz and E. Silverstein, Phys. Rev. D62 (2000) 045021, hep-th/0001206; it ibid., Phys. Rev. D62 (2000) 085003; R. Bousso and J. Polchinski, JHEP 0006 (2000) 006; J.L. Feng, J. March-Russell, S. Sethi and F. Wilczek, hep-th/0005276.

[10] D.J. Gross, J.A. Harvey, E. Martinec and R. Rohm, Phys. Rev. Lett 54 (1985) 502; Nucl. Phys. B256 (1985) 253; Nucl. Phys. B267 (1986) 75.

[11] H. Verlinde, "Holography and Compactification," Nucl. Phys. B580 (2000) 264, hepth/9906182; S. Giddings, S. Kachru and J. Polchinski, "Hierarchies from Fluxes in String Compactifications", hep-th/0105097.

[12] S. Sethi, C. Vafa and E. Witten, "Constraints on Low Dimensional String Compactifications," Nucl. Phys. B480 (1996) 213, hep-th/9606122.

[13] J. Maldacena, "The Large N Limit of Superconformal Field Theories and Supergravity," ATMP 2 (1998) 231, hep-th/9711200.

[14] S. Gubser, I. Klebanov, and A. Peet, "Entropy and Temperature of Black 3-Branes", Phys. Rev. D54 (1996) 3915, hep-th/9602135. 
[15] I. Klebanov, "Wordvolume Approach to Absorption by Nondilatonic Branes", Nucl. Phys. B496 (1997) 231; S. Gubser, I. Klebanov, A. Tseytlin, "String Theory and Classical Absorption by Three-Branes", Nucl. Phys. B499 (1997) 217.

[16] J. Lykken, R. Myers, J. Wang, "Gravity in a Box", JHEP 0009 (2000) 009.

[17] S. Gubser, I. Klebanov and A. Polyakov, "Gauge Theory Correlators from Noncritical String Theory," Phys. Lett. B428 (1998) 105, hep-th/9802109; E. Witten, "Anti-de Sitter Space and Holography," ATMP 2 (1998) 253, hep-th/9802150.

[18] V. Balasubramanian, P. Kraus and A. Lawrence, "Bulk vs Boundary Dynamics in Anti-de Sitter Space-Time," Phys. Rev. D59 (1999) 046003, hep-th/9805171.

[19] S. Dimopoulos, S. Kachru, N. Kaloper, A. Lawrence and E. Silverstein, "Small Numbers From Tunneling Between Brane Throats," hep-th/0104239.

[20] S. Dimopoulos, "LHC, SSC and the Universe", Phys. Lett. B246 (1990) 347; N. Arkani-Hamed, L.J. Hall, C. Kolda and H. Murayama, "A New Perspective on Cosmic Coincidence Problems", Phys. Rev. Lett. 85 (2000) 4434, astro-ph/0005111.

[21] Renyue Cen, "Decaying Cold Dark Matter Model and Small-Scale Power", astro$\mathrm{ph} / 0005206$

[22] D.N. Spergel and P.J. Steinhardt, "Observational Evidence for Selfinteracting Cold Dark Matter", Phys. Rev. Lett. 84 (2000) 3760, astro-ph/9909386.

[23] P.J.E. Peebles, "Fluid Dark Matter", astro-ph/0002495.

[24] S. Dimopoulos and D. Sutter, Nucl. Phys. B452 (1995) 496.

[25] E. Cremmer, S. Ferrara, L. Girardello, and A. Van Proyen, Phys. Lett. B116 (1982) 231; A. Chamseddine, R. Arnowitt, and P. Nath, Phys. Rev. Lett. 49 (1982) 970; R. Barbieri, S. Ferrara, and C. Savoy, Phys. Lett. B110 (1982) 343; L. J. Hall, J. Lykken, and S. Weinberg, Phys. Rev. D27(1983) 2359.

[26] H.P. Nilles, Phys. Lett. B115 (1982) 193; Nucl. Phys. B217 (1983) 366.

[27] M. Dine, R. Rohm, N. Seiberg, E. Witten, Phys. Lett. B156 (1985) 55.

[28] M. Dine, W. Fischler, Nucl. Phys. B204 (1982) 346; S. Dimopoulos and S. Raby, Nucl. Phys. B219 (1982) 479; J. Polchinski and L. Susskind, Phys. Rev. D26 (1982) 3661.

[29] M. Dine and A. Nelson, Phys. Rev. D48 (1993) 1277, hep-th/9303230.

[30] L. Randall and R. Sundrum, Nucl. Phys. B557 (1999) 79; G.F. Giudice, M.A. Luty, H. Murayama and R. Rattazzi, JHEP 9812 (1998) 027.

[31] D. E. Kaplan, G. D. Kribs and M. Schmaltz, Phys. Rev. D62, 035010 (2000); Z. Chacko, M. A. Luty, A. E. Nelson and E. Ponton, JHEP 0001, 003 (2000); M. Schmaltz and W. Skiba, Phys. Rev. D62, 095005 (2000); Phys. Rev. D62, 095004 (2000); D. E. Kaplan and G. D. Kribs, JHEP 0009, 048 (2000).

[32] E.A. Mirabelli and M.E. Peskin, "Transmission of Supersymmetry Breaking from a Four-Dimensional Boundary", Phys. Rev. D58 (1998) 065002, hep-th/9712214

[33] S. Dimopoulos, S. Raby and F. Wilczek, Phys. Rev. D24 (1981) 1681. 
[34] S. Kachru and E. Silverstein, "4d Conformal Field Theories and Strings on Orbifolds," Phys. Rev. Lett. 80 (1998) 4855, hep-th/9802183;

A. Lawrence, N. Nekrasov and C. Vafa, "On Conformal Field Theories in Four Dimensions," Nucl. Phys. B533 (1998) 199, hep-th/9803015;

J. Distler and F. Zamora, "Non-Supersymmetric Conformal Field Theories from Stable Anti-de Sitter Spaces," Adv. Theor. Math. Ph. 2 (1999) 1405, hep-th/9810206.

[35] I. Antoniadis, S. Dimopoulos and G. Dvali, "Millimeter Range Forces in Superstring Theories with Weak Scale Compactification", Nucl. Phys. B516 (1998) 70, hep-ph/9710204

[36] I. Antoniadis, S. Dimopoulos, A. Pomarol and M. Quiros, "Soft Masses in Theories with Supersymmetry Breaking by TeV Compactification", Nucl. Phys. B544 (1999) 503, hep-ph/9810410

[37] T. Gherghetta and A. Pomarol, "A Warped Supersymmetric Standard Model," hep$\mathrm{ph} / 0012378$.

[38] J. Wess and J. Bagger, Supersymmetry and Supergravity, 2nd. ed., Princeton University Press (1992).

[39] Y. Grossman and M. Neubert, "Neutrino Masses and Mixings in Nonfactorizable Geometry", Phys. Lett. B474 (2000) 361, hep-ph/9912408

[40] J. Ellis, C. Kounnas and D. Nanopoulos, Nucl. Phys. B247 (1984) 373.

[41] V. Balasubramanian, S.B. Giddings and A. Lawrence, "What do CFTs tell us about anti-de Sitter spacetimes?" JHEP 9903 (1999) 001; hep-th/9902052.

[42] I.R. Klebanov, "World volume approach to absorption by non-dilatonic branes," Nuc. Phys. B496 (1997) 231.

[43] V. Balasubramanian, P. Kraus, A. Lawrence and S. Trivedi, "Holographic probes of anti-de Sitter spacetimes," Phys. Rev. D59 (1999) 104021; hep-th/9808017.

[44] T. Banks, M.R. Douglas, G.T. Horowitz and E. Martinec, "AdS dynamics from conformal field theory," hep-th/9808016.

[45] J. Maldacena, unpublished; E. Witten, unpublished; S. Gubser, "AdS/CFT and Gravity," hep-th/9912001; S. Giddings, E. Katz and L. Randall, "Linearized Gravity in Brane Backgrounds," JHEP 0003 (2000) 023, hep-th/0002091; M. Duff and J. Liu, "On the Equivalence of the Maldacena and Randall-Sundrum Pictures," Phys. Rev. Lett. 85 (2000) 2052, hep-th/0003237; S. Giddings and E. Katz, "Effective Theories and Black Hole Production in Warped Compactifications," hep-th/0009176; N. Arkani-Hamed, M. Porrati and L. Randall, "Holography and Phenomenology," hepth/0012148; R. Rattazzi and A. Zaffaroni, "Comments on the Holographic Picture of the Randall-Sundrum Model," hep-th/0012248.

[46] P. Breitenlohner and D.Z. Freedman, "Positive energy in anti-de Sitter backgrounds and gauged extended supergravity," Phys. Lett. B115 (1982) 197; ibid., "Stability in gauged extended supergravity," Ann. Phys. NY 144 (1982) 249; L. Mezinescu and P.K. Townsend, "Stability at a local maximum in higher-dimensional anti-de Sitter space and applications to supergravity," Ann. Phys. NY 160 (1985) 406. 